\title{
Pathogenesis of coronary artery disease: focus on genetic risk factors and identification of genetic variants
}

This article was published in the following Dove Press journal:

The Application of Clinical Genetics

16 January 2014

Number of times this article has been viewed

\author{
Sergi Sayols-Baixeras \\ Carla Lluís-Ganella \\ Gavin Lucas \\ Roberto Elosua \\ Cardiovascular Epidemiology and \\ Genetics Research Group, Institut \\ Hospital del Mar d'Investigacions \\ Mèdiques, Barcelona, Spain
}

Correspondence: Roberto Elosua Cardiovascular Epidemiology and Genetics Research Group, Institut Hospital del Mar d'Investigacions Mèdiques, Barcelona, Spain Email relosua@imim.es

\begin{abstract}
Coronary artery disease (CAD) is the leading cause of death and disability worldwide, and its prevalence is expected to increase in the coming years. CAD events are caused by the interplay of genetic and environmental factors, the effects of which are mainly mediated through cardiovascular risk factors. The techniques used to study the genetic basis of these diseases have evolved from linkage studies to candidate gene studies and genome-wide association studies. Linkage studies have been able to identify genetic variants associated with monogenic diseases, whereas genome-wide association studies have been more successful in determining genetic variants associated with complex diseases. Currently, genome-wide association studies have identified approximately 40 loci that explain $6 \%$ of the heritability of CAD. The application of this knowledge to clinical practice is challenging, but can be achieved using various strategies, such as genetic variants to identify new therapeutic targets, personal genetic information to improve disease risk prediction, and pharmacogenomics. The main aim of this narrative review is to provide a general overview of our current understanding of the genetics of coronary artery disease and its potential clinical utility.
\end{abstract}

Keywords: coronary artery disease, pathogenesis, genetic risk factors, genetic variants

\section{Introduction}

Coronary artery disease (CAD) is the principal individual cause of mortality and morbidity worldwide. A recent report on the Global Burden of Disease, which proposes disability-adjusted life years (DALYs, calculated as the sum of years of life lost and years lived with disability) as a new metric to measure disease burden, indicates that CAD accounted for the largest proportion of DALYs due to a single cause worldwide in 2010, explaining $5 \%$ of the total number of DALYS (Figure 1). ${ }^{1}$

$\mathrm{CAD}$ is a complex chronic inflammatory disease, characterized by remodeling and narrowing of the coronary arteries supplying oxygen to the heart. It can have various clinical manifestations, including stable angina, acute coronary syndrome, and sudden cardiac death. It has a complex etiopathogenesis and a multifactorial origin related to environmental factors, such as diet, smoking, and physical activity, and genetic factors ${ }^{2}$ that modulate risk of the disease both individually and through interaction.

In this narrative review, we summarize the main etiopathogenic mechanisms that underlie $\mathrm{CAD}$, with a focus on current knowledge concerning the genetic architecture of the disease and the clinical utility of this knowledge. 


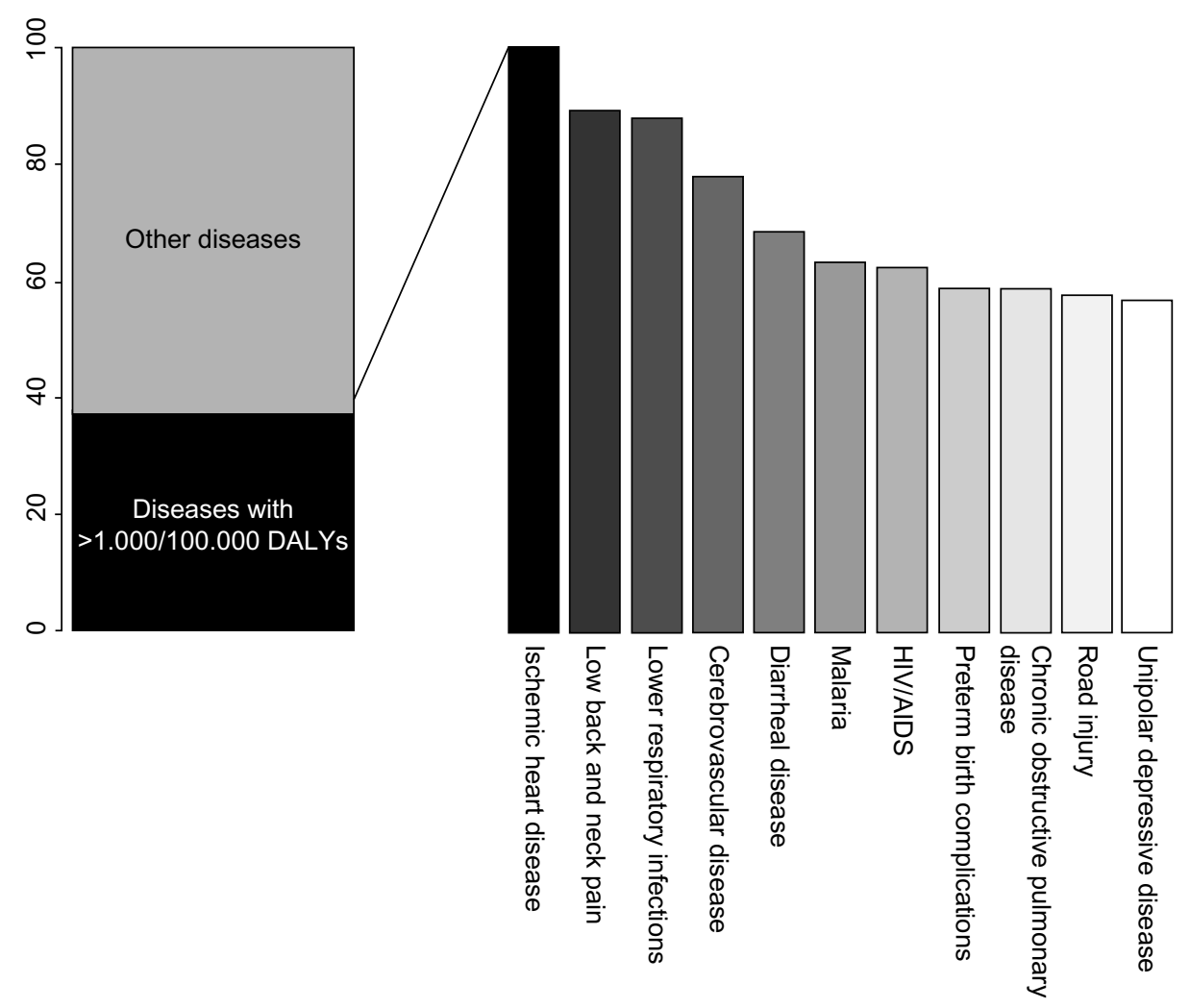

Figure I The top eleven diseases explain $37.7 \%$ of the global burden of disease measured as DALYs, with coronary artery disease as the leading cause of DALYs in 2010. Abbreviations: DALYs, disability-adjusted life years; AIDS, acquired immune deficiency syndrome; HIV, human immunodeficiency virus.

\section{Atherosclerosis, the main etiopathogenic mechanism of CAD}

Atherosclerosis is the main etiopathogenic process that causes $\mathrm{CAD}$, and its progression is related to an interplay between environmental and genetic factors, with the latter exerting their effects either directly or via cardiovascular risk factors (Figure 2). Although clinical ischemic cardiovascular events usually appear after the fifth decade of life in men and the sixth decade of life in women, this process starts early in life, even during fetal development. ${ }^{3}$

Briefly, atherosclerosis is a silent progressive chronic process characterized by accumulation of lipids, fibrous elements, and inflammatory molecules in the walls of the large arteries. ${ }^{48}$ This process begins with the efflux of lowdensity lipoprotein (LDL) cholesterol to the subendothelial space, which can then be modified and oxidized by various agents. Oxidized/modified LDL particles are potent chemotactic molecules that induce expression of vascular cell adhesion molecule and intercellular adhesion molecule at the endothelial surface, and promote monocyte adhesion and migration to the subendothelial space. Monocytes differentiate to macrophages in the intima media. Recently, different subsets of monocytes have been identified, and their roles appear to be different according to the phase of

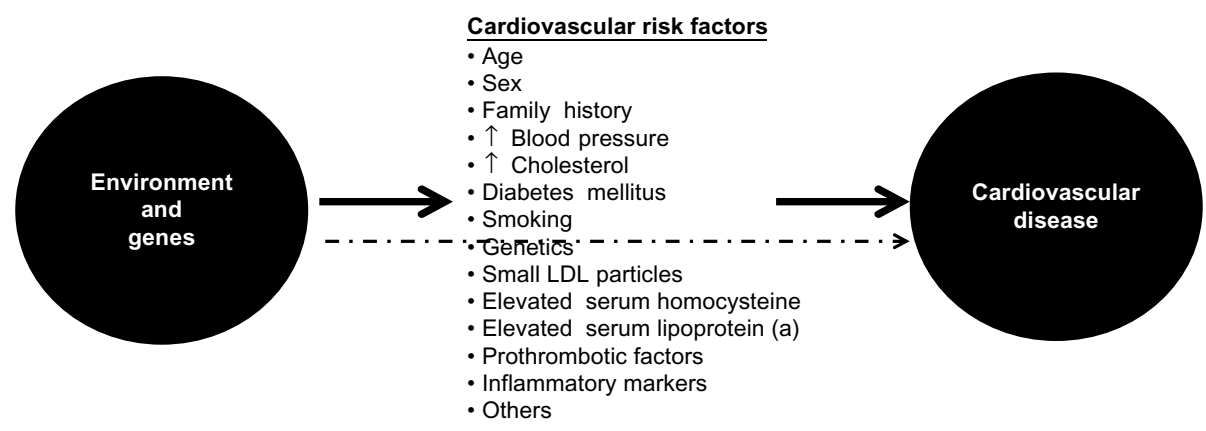

Figure 2 Genetic and environmental causes of development and progression of atherosclerosis act directly or through known intermediate traits. Abbreviation: LDL, low-density lipoprotein. 
atherosclerosis in which they are involved..$^{9}$ Macrophages bind oxidized LDL via scavenger receptors to become foam cells, ${ }^{5}$ and also have proinflammatory functions, including the release of cytokines such as interleukins and tumor necrosis factor. The final result of this process is formation of the first typical atherosclerotic lesion, ie, the fatty streak, in which foam cells are present in the subendothelial space.

Other types of leukocytes, such as lymphocytes and mast cells, also accumulate in the subendothelial space. ${ }^{10}$ The cross-talk between monocytes, macrophages, foam cells, and T-cells results in cellular and humoral immune responses, and ultimately in a chronic inflammatory state with the production of several proinflammatory molecules. ${ }^{11,12}$ This process continues with the migration of smooth muscle cells from the medial layer of the artery into the intima, resulting in the transition from a fatty streak to a more complex lesion. ${ }^{5}$ Once smooth muscle cells are in the intima media, they produce extracellular matrix molecules, creating a fibrous cap that covers the original fatty streak. Foam cells inside the fibrous cap die and release lipids that accumulate in the extracellular space, forming a lipid-rich pool known as the necrotic core. ${ }^{13}$ The result of this process is formation of the second atherosclerotic lesion, the fibrous plaque.

The thickness of the fibrous cap is key for maintaining the integrity of the atherosclerotic plaque, ${ }^{8}$ and two types of plaque can be defined depending on the balance between formation and degradation of this fibrous cap, ie, stable and unstable or vulnerable. Stable plaques have an intact, thick fibrous cap composed of smooth muscle cells in a matrix rich in type I and III collagen. ${ }^{14}$ Protrusion of this type of plaque into the lumen of the artery produces flow-limiting stenosis, leading to tissue ischemia and usually stable angina. Vulnerable plaques have a thin fibrous cap made mostly of type I collagen and few or no smooth muscle cells, but abundant macrophages and proinflammatory and prothrombotic molecules. ${ }^{8,10}$ These plaques are prone to erosion or rupture, exposing the core of the plaque to circulating coagulation proteins, causing thrombosis, sudden occlusion of the artery lumen, ${ }^{8,10}$ and usually an acute coronary syndrome. Intraplaque hemorrhage is also a potential contributor to progression of atherosclerosis, and appears to occur when the vasa vasorum invades the intima from the adventitia. ${ }^{15}$

\section{Study of the genetic architecture of disease}

In order to study the genetic factors associated with a disease, several sequential steps must be followed. The first step involves quantification of the genetic component of the disease, which can be expressed as its heritability, ie, the proportion of the total population variance of the phenotype at a particular time or age that is attributable to genetic variation. ${ }^{16}$ The heritability of some phenotypes associated with arteriosclerosis has already been determined, and generally ranges from $40 \%$ to $55 \%$ (Table 1 ). ${ }^{17,18}$

The second step is to study the genetic architecture of the disease, ie, identify the loci, and within these loci, the genetic variants that modulate disease susceptibility. However, this task is one of the greatest challenges in current genetic research. Depending on the observed patterns of inheritance, it is possible to classify genetic diseases in two broad classes, ie, monogenic or Mendelian diseases, in which genetic variation in one gene accounts for most or all of the variation in disease risk: ${ }^{19}$ and complex diseases, which are characterized by complex patterns of inheritance caused by the combination of multiple genetic variants (often with a small effect) and environmental factors, and modulated by their mutual interaction. ${ }^{20}$ For example, in the case of CAD, the effects of known genetic variants range from an odds ratio of 1.04 to approximately 1.30 per copy of the risk allele. ${ }^{21}$ Studies of the genetic architecture of a disease generally have two approaches, ie, linkage and association studies. ${ }^{17}$

\section{Linkage studies}

In these studies, large families with several affected and unaffected relatives across one or more generations are identified and recruited. ${ }^{22,23}$ Classically, large numbers of genetic markers, uniformly distributed throughout the genome, are analyzed to see if their transmission from generation to generation is associated with the presence of the disease (segregation). The initial objective is to identify regions of the genome that contain genes predisposing to or causing the disease under study. Thereafter, the chromosomal

Table I Main results of different studies analyzing the heritability of several phenotypes associated with arteriosclerosis

\begin{tabular}{|c|c|c|}
\hline Phenotype & Heritability & References \\
\hline \multicolumn{3}{|l|}{$\overline{C A D}$} \\
\hline $\begin{array}{l}\text { Acute myocardial } \\
\text { infarction }\end{array}$ & 0.56 & Nora et a ${ }^{89}$ \\
\hline Mortality from & $0.53-0.57$ (men) & Zdravkovic et al, ${ }^{90}$ \\
\hline CAD & 0.58 (women) & Wienke et $\mathrm{al}^{91}$ \\
\hline $\begin{array}{l}\text { Coronary artery } \\
\text { calcification }\end{array}$ & 0.42 & Peyser et $\mathrm{al}^{92}$ \\
\hline \multicolumn{3}{|l|}{ Atherosclerosis } \\
\hline $\begin{array}{l}\text { Carotid artery } \\
\text { atherosclerosis }\end{array}$ & $0.21-0.64$ & $\begin{array}{l}\text { Xiang et al, }{ }^{93} \text { Fox et al, }{ }^{94} \\
\text { Swan et al, }{ }^{95} \text { North et al, }{ }^{96} \\
\text { Hunt et al }{ }^{97}\end{array}$ \\
\hline
\end{tabular}

Abbreviation: CAD, coronary artery disease. 
region that segregates with the disease can be fine-mapped to identify the causal gene. ${ }^{17,22,23}$ This type of study has been successful in identifying many disease genes, particularly those that cause Mendelian traits, but less successful in identifying genes associated with complex diseases. ${ }^{24}$ In the case of CAD, notable successes include the identification of variants in $A L O X 5 A P$ as being associated with coronary and cerebrovascular diseases, ${ }^{25}$ in $M E F 2 A$ as being associated with $\mathrm{CAD},{ }^{26}$ and in $\mathrm{PCSK} 9$ as a gene for which variation is relevant in the metabolism of cholesterol. ${ }^{27}$

\section{Association studies}

Association studies are likely to be more effective tools than linkage studies for studying genetically complex traits because they can have greater statistical power to detect genetic variants with small effects. ${ }^{28}$ These types of studies evaluate the association between genetic variants (usually single nucleotide polymorphisms or copy number variations) and the presence/absence of a disease or a specific phenotype. The biggest challenges for this type of study are the accuracy of phenotype definition and replication of the findings. In order to identify genetic variants with small effects, large sample sizes are required, which are usually obtained by pooling different samples and populations, potentially with different phenotyping methods or criteria. In many cases, this heterogeneity results in dilution of the effects of causal genetic variants. In other cases, the phenotype itself may be difficult to define (eg, fibromyalgia) or show substantial intraindividual variability (eg, blood pressure), diluting the observable effect of the causal variant on the phenotype of interest. There are several types of association studies, as follows.

\section{Candidate gene studies}

Association studies in candidate genes, usually known to be related to intermediate traits, have been widely used for the study of complex diseases. ${ }^{4,29,30}$ This approach is based on an a priori hypothesis generated from knowledge of the disease pathogenesis or previous results. ${ }^{17,31}$ In the 1990 s, this type of research became very popular and many studies analyzing the relationship between genetic variants and phenotypes were published, although their main findings were often difficult to replicate. ${ }^{32}$

\section{Genome-wide association studies}

The goal of genome-wide association studies (GWAS) is to identify genetic variants associated with complex phenotypes without the need for prior selection of candidate loci or genes. ${ }^{33}$ GWAS are based on two assumptions: first, a large proportion of common variation in the genome can be captured by a relatively small number of genetic variants, an hypothesis that is supported by evidence from the HapMap project; ${ }^{34}$ and, second, common complex diseases are mainly caused by common genetic variants.

This type of study became possible through technologic advances that allowed large numbers of single nucleotide polymorphisms to be genotyped throughout the genome and common patterns of linkage disequilibrium in different populations to be determined, thanks to studies like the Human Genome Project and the HapMap Project. ${ }^{34,35}$ This evidence allowed the possibility of searching the human genome for common variants associated with a huge variety of phenotypes and diseases. ${ }^{36}$ Moreover, powerful association analysis methods and software have also been developed. ${ }^{37,38}$ These studies are hypothesis-free, and due to the multiple comparisons and the need to reduce the burden of potential type I errors, they have to correct the $P$-value to be considered statistically significant according to the number of tests performed. Usually, this statistical significance threshold is located at a $P$-value $<10^{-8}$.

In parallel, international collaborations and consortia have provided new insights in medical research. ${ }^{39}$ While the number of studies that use this methodology has increased rapidly in recent years (Figure 3), this type of design is known to have some limitations (Table 2). These include the low proportion of heritability explained by the genetic variants identified, which has been found to be lower than $10 \%$ for most phenotypes, ${ }^{4,40}$ and the fact that the results are based on statistical association and do not provide functional insights. However, these studies have consistently identified hundreds of loci in dozens of clinically important phenotypes, ${ }^{4,41}$ providing further insights into the genetics of complex diseases.

\section{Whole-genome sequencing studies}

The human genome contains approximately 3.1 billion nucleotides with approximately 56 million genetic variants. The exome, ie, the part of the genome formed by protein-coding exons, comprises approximately 30 million nucleotides and 23,500 genes. ${ }^{42}$ Rapidly improving whole-genome sequencing (WGS) technologies are creating new research avenues based on sequencing entire individuals, ${ }^{39,42}$ and the rapidly decreasing costs of WGS will soon allow this technology to be used for tackling the genetic architecture of disease. ${ }^{43}$ WGS are expected to contribute to better definition of the genetic basis of a range of phenotypes, responses to therapy, 


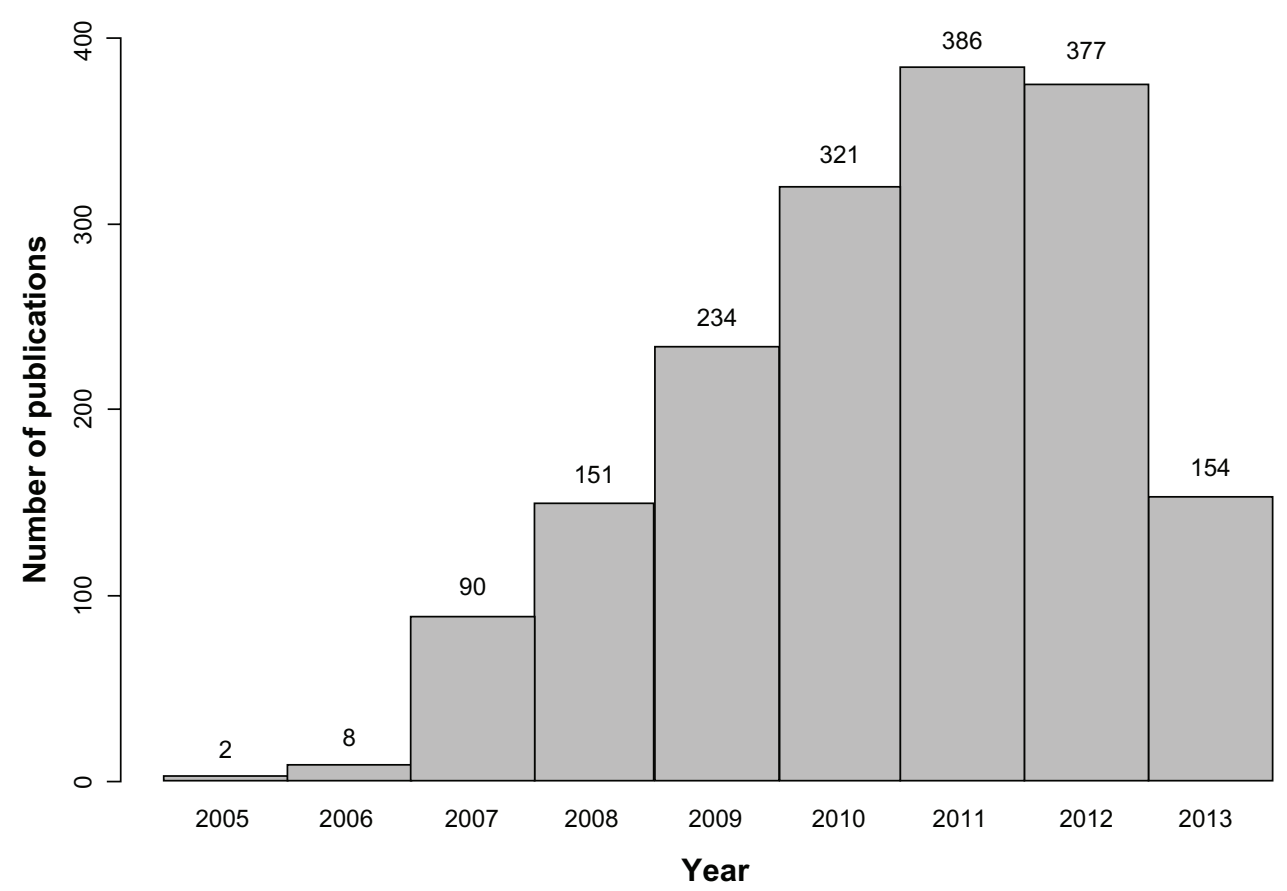

Figure 3 Number of articles published per year according to the genome-wide association studies catalog (accessed on September 27, 20I3).

and clinical outcomes. Although WGS mainly focus on Mendelian disorders, the WGS approach is becoming important for identifying and analyzing rare variants, which might have larger effects on disease risk than the common variants identified by GWAS. ${ }^{42}$

Table 2 Comparison between candidate gene studies and GWAS

\begin{tabular}{|c|c|c|}
\hline Feature & $\begin{array}{l}\text { Candidate } \\
\text { gene studies }\end{array}$ & $\begin{array}{l}\text { Genome-wide association } \\
\text { studies }\end{array}$ \\
\hline Hypothesis & $\begin{array}{l}\text { Need a priori } \\
\text { hypothesis }\end{array}$ & Hypothesis-free \\
\hline $\begin{array}{l}\text { Number of } \\
\text { genetic variants }\end{array}$ & $\begin{array}{l}\text { Limited (one } \\
\text { to hundreds) }\end{array}$ & $\begin{array}{l}\text { Large (hundreds of thousands } \\
\text { to millions, with imputation) }\end{array}$ \\
\hline Sample size & $\begin{array}{l}\text { Limited (usually } \\
\text { hundreds) }\end{array}$ & $\begin{array}{l}\text { Large (hundreds to hundreds } \\
\text { of thousands) }\end{array}$ \\
\hline \multirow[t]{4}{*}{ Biases } & Selection bias & Selection bias \\
\hline & Confounding & Confounding \\
\hline & $\begin{array}{l}\text { Population } \\
\text { stratification }\end{array}$ & $\begin{array}{l}\text { Population stratification } \\
\text { (methods to control) }\end{array}$ \\
\hline & Publication bias & \\
\hline \multirow[t]{4}{*}{ Limitations } & Sample size & Control for multiple testing \\
\hline & $\begin{array}{l}\text { Nonreplicability } \\
\text { of results }\end{array}$ & Phenotype definition \\
\hline & $\begin{array}{l}\text { Lack of } \\
\text { thoroughness }\end{array}$ & Based on common variants \\
\hline & $\begin{array}{l}\text { Low genetic } \\
\text { coverage }\end{array}$ & $\begin{array}{l}\text { Statistical versus functional } \\
\text { association }\end{array}$ \\
\hline False positive rate & Large & Low \\
\hline False negative rate & Low & Large \\
\hline
\end{tabular}

Note: Data summarized from many studies. ${ }^{28,98-104}$

Abbreviation: GWAS, genome-wide association studies.
One of the main disadvantages of WGS is the rate of false positive/false negative results in variant calling, and identification of the true causal genetic variant. Considering a false positive rate of $2 \%$, an analysis of three billion genetic variants per genome would yield 60,000,000 miscalled variants. Therefore, false positives are expected to remain a major limitation of WGS, and alternative methods for validating variants identified by this approach will be necessary. Also, WGS will have a real challenge in identifying the true causal genetic variants among all alleles because all genes and proteins carry several nonpathogenic variants. For this reason, a classification of genetic variants according to the strength of the evidence for causality has been proposed as follows: disease-causing, likely disease-causing, disease-associated, functional but not associated with disease; and unknown biological function. ${ }^{42}$

\section{Current knowledge of genetic architecture of CAD}

Our understanding of the genetic architecture of CAD has improved considerably since 2007 when the first GWAS of this disease were published. The first two studies were published simultaneously and identified the 9p21 locus to be associated with myocardial infarction ${ }^{44}$ and $\mathrm{CAD},{ }^{45}$ and a third study replicated these findings. ${ }^{46}$ At the beginning of 2013, a meta-analysis of several GWAS identified a final set of about 40 genetic variants associated with CAD (Table 3) 
Table 3 Summary of main findings of most recent meta-analysis of genome-wide association studies in coronary artery disease, showing the lead single nucleotide polymorphism of each locus, the closest gene, chromosomal location, risk allele and frequency, $P$-value, and effect size of the reported associations

\begin{tabular}{|c|c|c|c|c|c|}
\hline rsID & Gene located at or near loci & Chr & $\begin{array}{l}\text { Risk/nonrisk allele } \\
\text { (risk allele frequency) }\end{array}$ & $\begin{array}{l}\text { Combined } \\
\text { P-value }\end{array}$ & $\begin{array}{l}\text { Combined } \\
\text { OR }\end{array}$ \\
\hline rs602633 & SORTI & I & $\mathrm{C} / \mathrm{A}(0.77)$ & $1.47 \times 10^{-25}$ & 1.12 \\
\hline rs|7|I4036 & PPAP2B & 1 & A/G (0.9I) & $5.80 \times 10^{-12}$ & 1.11 \\
\hline rs4845625 & IL6R & 1 & $\mathrm{~T} / \mathrm{C}(0.47)$ & $3.64 \times 10^{-10}$ & 1.09 \\
\hline rs67258870 & WDR $/ 2$ & 2 & $\mathrm{C} / \mathrm{T}(0.1 \mathrm{I})$ & $1.16 \times 10^{-15}$ & 1.12 \\
\hline rs5I5I35 & $A P O B$ & 2 & $\mathrm{G} / \mathrm{A}(0.83)$ & $2.56 \times 10^{-10}$ & 1.03 \\
\hline rs225264I & ZEB2-ACO74093 & 2 & $\mathrm{G} / \mathrm{A}(0.46)$ & $5.30 \times 10^{-8}$ & 1.06 \\
\hline rs 1561198 & VAMP5-VAMP8-GGCX & 2 & A/G (0.45) & $1.22 \times 10^{-10}$ & 1.07 \\
\hline rs65447I3 & ABCG5-ABCG8 & 2 & $\mathrm{~T} / \mathrm{C}(0.30)$ & $2.12 \times 10^{-9}$ & 0.96 \\
\hline rs9818870 & MRAS & 3 & $\mathrm{~T} / \mathrm{C}(0.14)$ & $2.62 \times 10^{-9}$ & 1.07 \\
\hline rs7692387 & GUCYIA3 & 4 & $\mathrm{G} / \mathrm{A}(0.8 \mathrm{I})$ & $2.65 \times 10^{-11}$ & 1.13 \\
\hline rs 1878406 & EDNRA & 4 & $\mathrm{~T} / \mathrm{C}(0.15)$ & $2.54 \times 10^{-8}$ & 1.09 \\
\hline rs273909 & SLC22A4-SLC22A5 & 5 & $\mathrm{C} / \mathrm{T}(0.14)$ & $9.62 \times 10^{-10}$ & 1.11 \\
\hline rs 12190287 & TCF2I & 6 & $\mathrm{C} / \mathrm{G}(0.59)$ & $4.94 \times 10^{-13}$ & 1.07 \\
\hline rs2048327 & SLC22A3-LPAL2-LPA & 6 & $\mathrm{G} / \mathrm{A}(0.35)$ & $6.86 \times 10^{-11}$ & 1.06 \\
\hline rs9369640 & PHACTRI & 6 & $\mathrm{~A} / \mathrm{C}(0.65)$ & $7.53 \times 10^{-22}$ & 1.09 \\
\hline rs 10947789 & KCKN5 & 6 & $\mathrm{~T} / \mathrm{C}(0.76)$ & $9.81 \times 10^{-9}$ & 1.01 \\
\hline rs4252I20 & PLG & 6 & $\mathrm{~T} / \mathrm{C}(0.73)$ & $4.88 \times 10^{10}$ & 1.07 \\
\hline rsII556924 & $\mathrm{ZC} 3 \mathrm{HCl}$ & 7 & $\mathrm{C} / \mathrm{T}(0.65)$ & $6.74 \times 10^{-17}$ & 1.09 \\
\hline rs2023938 & HDAC9 & 7 & $\mathrm{G} / \mathrm{A}(0.10)$ & $4.94 \times 10^{-8}$ & 1.13 \\
\hline rs264 & $L P L$ & 8 & $\mathrm{G} / \mathrm{A}(0.86)$ & $2.88 \times 10^{-9}$ & 1.06 \\
\hline rs2954029 & TRIBI & 8 & $\mathrm{~A} / \mathrm{T}(0.55)$ & $4.75 \times 10^{-9}$ & 1.05 \\
\hline rs1333049 & CDKN2BASI & 9 & C/G (0.47) & $1.39 \times 10^{-52}$ & 1.23 \\
\hline rs3217992 & & 9 & A/G (0.38) & $7.75 \times 10^{-57}$ & 1.16 \\
\hline rs579459 & $A B O$ & 9 & $\mathrm{C} / \mathrm{T}(0.2 \mathrm{I})$ & $2.66 \times 10^{-8}$ & 1.07 \\
\hline rs 12413409 & CYPI 7AI-CNNM2-NT5C2 & 10 & G/A (0.89) & $6.26 \times 10^{-8}$ & 1.10 \\
\hline rs2505083 & KIAA I 462 & 10 & $\mathrm{C} / \mathrm{T}(0.42)$ & $1.35 \times 10^{-11}$ & 1.06 \\
\hline rs50II20 & CXCLI2 & 10 & A/G (0.83) & $1.79 \times 10^{-9}$ & 1.07 \\
\hline rs2047009 & & 10 & $\mathrm{C} / \mathrm{A}(0.48)$ & $1.59 \times 10^{-9}$ & 1.05 \\
\hline rs 974819 & PDGFD & 11 & A/G (0.29) & $3.55 \times 10^{-11}$ & 1.07 \\
\hline rs3184504 & $\mathrm{SH} 2 \mathrm{~B} 3$ & 12 & $\mathrm{~T} / \mathrm{C}(0.40)$ & $5.44 \times 10^{-11}$ & 1.07 \\
\hline rs4773| 44 & COL4AI-COL4A2 & 13 & $\mathrm{G} / \mathrm{A}(0.42)$ & $1.43 \times 10^{-11}$ & 1.07 \\
\hline rs95I5203 & & 13 & $\mathrm{~T} / \mathrm{C}(0.74)$ & $5.85 \times 10^{-12}$ & 1.08 \\
\hline rs9319428 & FLTI & 13 & A/G (0.32) & $7.32 \times 10^{-11}$ & 1.10 \\
\hline rs28958II & HHIPLI & 14 & $\mathrm{C} / \mathrm{T}(0.43)$ & $4.08 \times 10^{-10}$ & 1.06 \\
\hline rs7173743 & ADAMTS7 & 15 & $\mathrm{~T} / \mathrm{C}(0.58)$ & $6.74 \times 10^{-13}$ & 1.07 \\
\hline rs|75|4846 & FURIN-FES & 15 & $\mathrm{~A} / \mathrm{C}(0.44)$ & $9.33 \times 10^{-11}$ & 1.04 \\
\hline rs|2936587 & RAII-PEMT-RASDI & 17 & G/A (0.59) & $1.24 \times 10^{-9}$ & 1.06 \\
\hline rs228I727 & SMG6 & 17 & $\mathrm{C} / \mathrm{T}(0.36)$ & $7.83 \times 10^{-9}$ & 1.05 \\
\hline rsl| 22608 & $L D L R$ & 19 & $\mathrm{G} / \mathrm{T}(0.76)$ & $6.33 \times 10^{-14}$ & 1.10 \\
\hline rs998260I & Gene desert (KCNE2) & 21 & $\mathrm{~T} / \mathrm{C}(0.13)$ & $7.67 \times 10^{-17}$ & 1.13 \\
\hline
\end{tabular}

Abbreviations: Chr, chromosome; OR, odds ratio.

that explains approximately $6 \%$ of the heritability of CAD. ${ }^{21}$ Some of these variants are related to lipid metabolism, blood pressure, and inflammation, which confirms the importance of these pathways in the pathogenesis of CAD. ${ }^{21}$ In contrast, this study found no overlap between these CAD loci and those associated with type 2 diabetes or glucometabolic traits. Moreover, most of these CAD loci are located in intergenic regions, or in regions with unknown function or where the relationship to atherosclerosis or its intermediate traits is unknown.

\section{Genetics of cardiovascular risk factors}

Classical cardiovascular risk factors, such as hypertension, diabetes, dyslipidemia, and obesity, are also considered to be complex traits caused by the interplay between genetic and environmental factors, as in the case of CAD. The GWAS 
approach has had a similar degree of success in identifying the genetic architecture of these risk factors and that of $\mathrm{CAD}$, in that only a small fraction of the heritability of these phenotypes has been explained (Table 4). ${ }^{47-53}$ While some of these genetic variants are also associated with CAD risk, others are not, ie, they have such small effects that very sample sizes would be required to detect them.

\section{Clinical utility of genetic knowledge}

The identification of genetic variants associated with disease has allowed us to improve our understanding of its pathogenesis, and ultimately to reduce the burden of disease at both the individual and population levels. Information derived from genetic studies could potentially help to reduce the burden of disease in three main ways, ie, the identification of new pharmacologic targets, improvements in identification of high-risk individuals, and pharmacogenomics.

\section{Identification of new pharmacologic targets}

Genetic studies can shed light on new metabolic pathways associated with the development and progression of atherosclerosis, and provide clues for identifying new pharmacologic targets. The following two examples illustrate the promise as well as the potential difficulties of this field.

\section{PCSK 9}

A clear example of the success of genetic studies in identifying molecules that may become new therapeutic targets is the PCSK9 gene. This gene was initially discovered by linkage studies to be associated with autosomal dominant hypercholesterolemia, ${ }^{27}$ for which new causal mutations were identified in $2003 .{ }^{54}$ The PCSK9 protein is crucial for metabolism of LDL cholesterol through its role in degradation of the LDL receptor, such that inhibition of this protein could become a viable treatment for hypercholesterolemia. ${ }^{55}$ Recent clinical trials in patients with primary hypercholesterolemia have shown that combination treatment with REGN727/SAR236553, a human monoclonal antibody to PCSK9, and either $10 \mathrm{mg}$ or $80 \mathrm{mg}$ of atorvastatin resulted in significantly greater reduction of LDL cholesterol than that obtained by $80 \mathrm{mg}$ of atorvastatin alone. ${ }^{56}$

\section{9p2I region}

The genetic variants associated with CAD at the 9p21 locus, which has been the top hit in all CAD GWAS since 2007, lie in an intergenic region close to a cluster of cell-cycle regulating tumor suppressor genes (CDKN2A and $C D K N 2 B)$ that overlap with a nonprotein coding RNA ( $C D K N 2 B A S$ or $A N R I L)$. While various hypotheses have been proposed to explain the functional basis of this association, the mechanism remains unclear, ${ }^{39,57}$ and this has prevented the identification of a therapeutic target.

\section{Improved identification of high-risk individuals}

In the case of CAD, primary prevention strategies in healthy asymptomatic individuals are very important because the first clinical manifestation of the disease is often catastrophic (MI or sudden death). Two main prevention strategies can be defined: the population approach, based on public health policies that affect the whole population, such as smoking bans $;{ }^{58}$ and the approach that targets high-risk individuals, based on implementing intensive preventive treatment in individuals at high risk of having the disease, based on their cardiovascular risk factor profile. ${ }^{59}$ Two main screening strategies are usually undertaken to identify high-risk individuals, ie, opportunistic screening and high-risk screening. In opportunistic screening, evaluation of cardiovascular risk factors and estimation of CAD risk is carried out in all individuals who come into contact with the health care system for any reason. Risk functions are the most commonly used method for estimating individual risk of having a CAD event, usually for a 10 -year period. ${ }^{59-61}$ Risk functions are mathematical equations that estimate the probability of developing CAD/ cardiovascular disease using information about cardiovascular risk factors that are strongly and independently related to CAD and can be evaluated by simple procedures in the laboratory or doctor's office.

Depending on their estimated risk, it is possible to categorize individuals into different risk categories (low, intermediate, high, and very high), and these categories are used to determine the intensity of preventive cardiovascular measures to be applied, which may range from lifestyle recommendations to prescription of drugs with various clinical objectives. Although risk functions can accurately predict the numbers of events that will occur in each risk category, many CAD events occur in individuals whose risk is too low to justify intensive treatment. ${ }^{62}$ For this reason, considerable effort has been invested in improving the classification of these intermediate-risk individuals into more appropriate risk categories.

Several biomarkers, including genetic variants, have been analyzed as candidates for improving the predictive capacity of risks functions. ${ }^{63}$ The main advantage of genetic variants is that they remain invariable throughout life, so it 
Table 4 Summary of main findings of most recent meta-analyses of genome-wide association studies of cardiovascular risk factors, showing the lead single nucleotide polymorphism of each locus, the closest gene, chromosomal location, risk allele and frequency, and $P$-value of the reported associations

\begin{tabular}{|c|c|c|c|c|c|c|}
\hline rsID & Loci & Chr & $\begin{array}{l}\text { Risk allele (Risk } \\
\text { allele frequency) }\end{array}$ & Combined $P$-value & $\begin{array}{l}\text { Known effect } \\
\text { on CAD }\end{array}$ & Reference \\
\hline \multicolumn{7}{|l|}{ Obesity } \\
\hline rs28I5752 & NEGRI & I & $A(0.6 I)$ & $1.61 \times 10^{-22}$ & No & 50 \\
\hline rs543874 & SECI6B & I & $\mathrm{G}(0.19)$ & $3.56 \times 10^{-23}$ & No & 50 \\
\hline rsI5I4I75 & TNNI3K & I & $\mathrm{A}(0.43)$ & $8.16 \times 10^{-14}$ & No & 50 \\
\hline rsI555543 & РTBP2 & I & $C(0.59)$ & $3.68 \times 10^{-10}$ & No & 50 \\
\hline rs984222 & TBXI5-WARS2 & I & $\mathrm{G}(0.64)$ & $3.81 \times 10^{-14}$ & No & 49 \\
\hline rs2867/25 & TMEM I 8 & 2 & $C(0.83)$ & $2.77 \times 10^{-49}$ & No & 50 \\
\hline rs7|3586 & RBJ & 2 & $C(0.47)$ & $6.17 \times 10^{-22}$ & No & 50 \\
\hline rs8879/2 & FANCL & 2 & $\mathrm{~T}(0.29)$ & $1.79 \times 10^{-12}$ & No & 50 \\
\hline rsI0195252 & GRB $/ 4$ & 2 & $\mathrm{~T}(0.60)$ & $2.09 \times 10^{-24}$ & No & 49 \\
\hline rs|3078807 & $L R P I B$ & 2 & $C(0.18)$ & $1.35 \times 10^{-10}$ & No & 50 \\
\hline rs9816226 & ETV5 & 3 & $\mathrm{~T}(0.82)$ & $1.69 \times 10^{-18}$ & No & 50 \\
\hline rs|3078807 & CADM2 & 3 & $G(0.20)$ & $3.94 \times 10^{-11}$ & No & 50 \\
\hline rs6795735 & ADAMTS9 & 3 & $C(0.60)$ & $9.79 \times 10^{-14}$ & No & 49 \\
\hline rs6784615 & NISCH-STABI & 3 & $\mathrm{~T}(0.94)$ & $3.84 \times 10^{-10}$ & No & 49 \\
\hline rs|3107325 & SLC39A8 & 4 & $\mathrm{~T}(0.07)$ & $1.50 \times 10^{-13}$ & No & 50 \\
\hline rsI0938397 & GNPDA2 & 4 & G (0.43) & $3.78 \times 10^{-31}$ & No & 50 \\
\hline rs21II2347 & FLJ35779 & 5 & $\mathrm{~T}(0.63)$ & $2.17 \times 10^{-13}$ & No & 50 \\
\hline rs4836133 & ZNF608 & 5 & $\mathrm{~A}(0.48)$ & $1.97 \times 10^{-9}$ & No & 50 \\
\hline rs68168I & CPEB4 & 5 & $\mathrm{~A}(0.34)$ & $1.91 \times 10^{-9}$ & No & 49 \\
\hline rs987237 & TFAP2B & 6 & $G(0.18)$ & $2.90 \times 10^{-20}$ & No & 50 \\
\hline rs206936 & NUDT3 & 6 & $G(0.21)$ & $3.02 \times 10^{-8}$ & No & 50 \\
\hline rs9491696 & RSPO3 & 6 & G (0.48) & $1.84 \times 10^{-40}$ & No & 49 \\
\hline rs6905288 & VEGFA & 6 & $A(0.56)$ & $5.88 \times 10^{-25}$ & No & 49 \\
\hline rsI29442I & LY86 & 6 & $G(0.6 I)$ & $1.75 \times 10^{-17}$ & No & 49 \\
\hline rsI055I44 & NFE2L3 & 7 & $\mathrm{~T}(0.2 \mathrm{I})$ & $9.97 \times 10^{-18}$ & No & 49 \\
\hline rsI0968576 & LRRN6C & 9 & $G(0.3 I)$ & $2.65 \times 10^{-13}$ & No & 50 \\
\hline rs10767664 & $B N F$ & II & $A(0.78)$ & $4.69 \times 10^{-26}$ & No & 50 \\
\hline rs3817334 & MTCH2 & 11 & $\mathrm{~T}(0.40)$ & $1.59 \times 10^{-12}$ & No & 50 \\
\hline rs4929949 & RPL27A & II & $C(0.52)$ & $2.80 \times 10^{-9}$ & No & 50 \\
\hline rs7। 38803 & FAIM2 & 12 & $A(0.38)$ & $1.82 \times 10^{-17}$ & No & 50 \\
\hline rs7|83|4 & ITPR2-SSPN & 12 & $\mathrm{G}(0.26)$ & $1.14 \times 10^{-17}$ & No & 50 \\
\hline rs 1443512 & $\mathrm{HOXCl} 3$ & 12 & $A(0.24)$ & $6.38 \times 10^{-17}$ & No & 49 \\
\hline rs477II22 & MTIF3 & 13 & $\mathrm{G}(0.24)$ & $9.48 \times 10^{-10}$ & No & 50 \\
\hline rsI0I50332 & NRXN3 & 14 & $C(0.21)$ & $2.75 \times 10^{-11}$ & No & 50 \\
\hline rsII847697 & PRKDI & 14 & $\mathrm{~T}(0.04)$ & $5.76 \times 10^{-11}$ & No & 50 \\
\hline rs224I423 & MAP2K5 & 15 & G (0.78) & $1.19 \times 10^{-18}$ & No & 50 \\
\hline rsI558902 & FTO & 16 & $\mathrm{~A}(0.42)$ & $4.8 \times 10^{-120}$ & No & 50 \\
\hline rs7359397 & $\mathrm{SH} 2 \mathrm{BI}$ & 16 & $\mathrm{~T}(0.40)$ & $1.88 \times 10^{-20}$ & No & 50 \\
\hline rs 12444979 & GPRC5B & 16 & $C(0.87)$ & $2.91 \times 10^{-21}$ & No & 50 \\
\hline rs571312 & $M C 4 R$ & 18 & $\mathrm{~A}(0.24)$ & $6.43 \times 10^{-42}$ & No & 50 \\
\hline rs2994I & KCTD $/ 5$ & 19 & $\mathrm{G}(0.67)$ & $3.01 \times 10^{-9}$ & No & 50 \\
\hline rs2287019 & QPCTL & 19 & $C(0.80)$ & $1.88 \times 10^{-16}$ & No & 50 \\
\hline rs3810291 & TMEMI 60 & 19 & $\mathrm{~A}(0.67)$ & $1.64 \times 10^{-12}$ & No & 50 \\
\hline rs4823006 & ZNRF3-KREMENI & 22 & $\mathrm{~A}(0.57)$ & $1.10 \times 10^{-11}$ & No & 49 \\
\hline \multicolumn{7}{|l|}{ Diabetes } \\
\hline rs340874 & PROXI & I & $C(0.52)$ & $6.6 \times 10^{-12}$ & No & 47 \\
\hline rs560887 & G6PC2 & 2 & $C(0.70)$ & $8.7 \times 10^{-218}$ & No & 47 \\
\hline rs780094 & GCKR & 2 & $C(0.62)$ & $5.6 \times 10^{-38}$ & No & 47 \\
\hline rs24302I & $B C L I I A$ & 2 & $\mathrm{~A}(0.46)$ & $2.9 \times 10^{-15}$ & No & 52 \\
\hline rs7578326 & IRSI & 2 & $A(0.64)$ & $5.4 \times 10^{-20}$ & No & 52 \\
\hline
\end{tabular}


Table 4 (Continued)

\begin{tabular}{|c|c|c|c|c|c|c|}
\hline rsID & Loci & Chr & $\begin{array}{l}\text { Risk allele (Risk } \\
\text { allele frequency) }\end{array}$ & Combined $P$-value & $\begin{array}{l}\text { Known effect } \\
\text { on CAD }\end{array}$ & Reference \\
\hline rs II708067 & $A D C Y 5$ & 3 & $\mathrm{~A}(0.78)$ & $7.1 \times 10^{-22}$ & No & 47 \\
\hline rsll 1920090 & $S L C 2 A 2$ & 3 & $\mathrm{~T}(0.87)$ & $8.1 \times 10^{-13}$ & No & 47 \\
\hline rs4457053 & ZBED3 & 5 & $G(0.26)$ & $2.8 \times 10^{-12}$ & No & 52 \\
\hline rs46075I7 & GCK & 7 & $A(0.16)$ & $6.5 \times 10^{-92}$ & No & 47 \\
\hline rs2191349 & DGKB-TMEM/95 & 7 & $\mathrm{~T}(0.52)$ & $3.0 \times 10^{-44}$ & No & 47 \\
\hline rs972283 & KLFI4 & 7 & $\mathrm{G}(0.55)$ & $2.2 \times 10^{-10}$ & No & 52 \\
\hline rs|l55847| & SLC30A8 & 8 & $A(0.68)$ & NA & No & 47 \\
\hline rs896854 & TP53INPI & 8 & $\mathrm{~T}(0.48)$ & $9.9 \times 10^{-10}$ & No & 52 \\
\hline rs7034200 & GLIS3 & 9 & $\mathrm{~A}(0.49)$ & $1.0 \times 10^{-12}$ & No & 47 \\
\hline rs 13292136 & $\mathrm{CHCHD} 9$ & 9 & $C(0.93)$ & $2.8 \times 10^{-8}$ & No & 52 \\
\hline rs 10885122 & ADRA2A & 10 & $\mathrm{G}(0.87)$ & $2.9 \times 10^{-16}$ & No & 47 \\
\hline rs4506565 & TCF7L2 & 10 & $\mathrm{~T}(0.3 \mathrm{I})$ & NA & No & 47 \\
\hline rs 10830963 & MTNRIB & 11 & $\mathrm{G}(0.30)$ & $5.8 \times 10^{-175}$ & No & 47 \\
\hline rs7944584 & $M A D D$ & 11 & $\mathrm{~A}(0.75)$ & $2.0 \times 10^{-18}$ & No & 47 \\
\hline rs 174550 & FADSI & 11 & $\mathrm{~T}(0.64)$ & $1.7 \times 10^{-15}$ & No & 47 \\
\hline rsII605924 & CRY2 & 11 & A (0.49) & $1.0 \times 10^{-14}$ & No & 47 \\
\hline$r s 231362$ & KCNQI & 11 & $\mathrm{G}(0.52)$ & $2.8 \times 10^{-13}$ & No & 52 \\
\hline rsI552224 & CENTD2 & 11 & $\mathrm{~A}(0.88)$ & $1.4 \times 10^{-22}$ & No & 52 \\
\hline rsI387I53 & MTNRIB & 11 & $\mathrm{~T}(0.28)$ & $7.8 \times 10^{-15}$ & No & 52 \\
\hline rsI53I343 & HMGA2 & 12 & $C(0.10)$ & $3.6 \times 10^{-9}$ & No & 52 \\
\hline rs7957।97 & HNFIA & 12 & $\mathrm{~T}(0.85)$ & $2.4 \times 10^{-8}$ & No & 52 \\
\hline rsII634397 & ZFAND6 & 15 & $G(0.60)$ & $2.4 \times 10^{-9}$ & No & 52 \\
\hline rsII07|657 & $C 2 C D 4 B$ & 15 & $A(0.63)$ & $3.6 \times 10^{-8}$ & No & 47 \\
\hline rs8042680 & PRCI & 15 & $A(0.22)$ & $2.4 \times 10^{-10}$ & No & 52 \\
\hline rs5945326 & DUSPQ & $x$ & $A(0.79)$ & $3.0 \times 10^{-10}$ & No & 52 \\
\hline \multicolumn{7}{|c|}{ Total cholesterol } \\
\hline rs $12027 \mid 35$ & LDLRAPI & I & $\mathrm{T}(0.45)$ & $4 \times 10^{-11}$ & Yes & 51 \\
\hline rs75I5577 & EVI5 & I & $A(0.2 I)$ & $3 \times 10^{-8}$ & No & 51 \\
\hline rs2642442 & $\mathrm{MOSCl}$ & I & $\mathrm{T}(0.32)$ & $6 \times 10^{-13}$ & No & 51 \\
\hline rs514230 & IRF2BP2 & I & $\mathrm{T}(0.48)$ & $5 \times 10^{-14}$ & No & 51 \\
\hline rs757097| & RAB3GAPI & 2 & $C(0.34)$ & $2 \times 10^{-8}$ & No & 51 \\
\hline rs2290I59 & RAFI & 3 & $\mathrm{G}(0.22)$ & $4 \times 10^{-9}$ & No & 51 \\
\hline rs 12916 & $H M G C R$ & 5 & $\mathrm{~T}(0.39)$ & $9 \times 10^{-47}$ & No & 51 \\
\hline rs6882076 & TIMD4 & 5 & $C(0.35)$ & $7 \times 10^{-28}$ & No & 51 \\
\hline rs3177928 & HLA & 6 & $G(0.16)$ & $4 \times 10^{-19}$ & No & 51 \\
\hline rs28I4982 & C6orflo6 & 6 & $C(0.11)$ & $5 \times 10^{-11}$ & No & 51 \\
\hline rs9488822 & FRK & 6 & $A(0.35)$ & $2 \times 10^{-10}$ & No & 51 \\
\hline rs 12670798 & DNAHII & 7 & $\mathrm{~T}(0.23)$ & $9 \times 10^{-10}$ & No & 51 \\
\hline rs2072183 & NPCILI & 7 & $G(0.25)$ & $3 \times 10^{-11}$ & No & 51 \\
\hline rs208I687 & CYP7AI & 8 & $C(0.35)$ & $2 \times 10^{-8}$ & No & 51 \\
\hline rs2737229 & TRPSI & 8 & $A(0.30)$ & $2 \times 10^{-8}$ & No & 51 \\
\hline rs2255I4I & GPAM & 10 & $\mathrm{G}(0.30)$ & $2 \times 10^{-10}$ & No & 51 \\
\hline rsI01287II & SPTY2DI & II & $C(0.28)$ & $3 \times 10^{-8}$ & No & 51 \\
\hline rs794I030 & UBASH3B & II & $C(0.38)$ & $2 \times 10^{-10}$ & No & 51 \\
\hline rsII065987 & BRAP & 12 & $\mathrm{~A}(0.42)$ & $7 \times 10^{-12}$ & No & 51 \\
\hline rsll 69288 & HNFIA & 12 & $\mathrm{~A}(0.33)$ & $1 \times 10^{-14}$ & No & 51 \\
\hline rs2000999 & $H P R$ & 16 & $G(0.20)$ & $3 \times 10^{-24}$ & No & 51 \\
\hline rs4420638 & CILP2 & 19 & $\mathrm{~T}(0.07)$ & $3 \times 10^{-38}$ & No & 51 \\
\hline rs 492602 & FLJ36070 & 19 & A (0.49) & $2 \times 10^{-10}$ & No & 51 \\
\hline rs2277862 & ERGIC3 & 20 & $C(0.15)$ & $4 \times 10^{-10}$ & No & 51 \\
\hline rs2902940 & MAFB & 20 & $\mathrm{~A}(0.29)$ & $6 \times 10^{-11}$ & No & 51 \\
\hline \multicolumn{7}{|l|}{ Triglycerides } \\
\hline rs2131925 & ANGPTL3 & I & $\mathrm{T}(0.32)$ & $9 \times 10^{-43}$ & No & 51 \\
\hline
\end{tabular}


Table 4 (Continued)

\begin{tabular}{|c|c|c|c|c|c|c|}
\hline rsID & Loci & Chr & $\begin{array}{l}\text { Risk allele (Risk } \\
\text { allele frequency) }\end{array}$ & Combined P-value & $\begin{array}{l}\text { Known effect } \\
\text { on CAD }\end{array}$ & Reference \\
\hline rsI042034 & $A P O B$ & 2 & $\mathrm{~T}(0.22)$ & $1 \times 10^{-45}$ & Yes & 51 \\
\hline rs 1260326 & GCKR & 2 & $C(0.4 I)$ & $6 \times 10^{-133}$ & No & 51 \\
\hline rs10195252 & COBLLI & 2 & $\mathrm{~T}(0.40)$ & $2 \times 10^{-10}$ & No & 51 \\
\hline rs645040 & MSL2LI & 3 & $\mathrm{~T}(0.22)$ & $3 \times 10^{-8}$ & No & 51 \\
\hline rs442I77 & KLHL8 & 4 & $\mathrm{~T}(0.4 \mathrm{I})$ & $9 \times 10^{-12}$ & No & 51 \\
\hline rs968666I & MAP3KI & 5 & $C(0.20)$ & $1 \times 10^{-10}$ & No & 51 \\
\hline rs2247056 & HLA & 6 & $C(0.25)$ & $2 \times 10^{-15}$ & No & 51 \\
\hline rs|3238203 & TYWIB & 7 & $C(0.04)$ & $1 \times 10^{-9}$ & No & 51 \\
\hline rs17|45738 & $M L X I P L$ & 7 & $C(0.12)$ & $6 \times 10^{-58}$ & No & 51 \\
\hline rsII776767 & PINXI & 8 & $\mathrm{G}(0.37)$ & $1 \times 10^{-8}$ & No & 51 \\
\hline rs|49574| & NAT2 & 8 & $\mathrm{~A}(0.22)$ & $5 \times 10^{-14}$ & No & 51 \\
\hline rs 12678919 & $L P L$ & 8 & $A(0.12)$ & $2 \times 10^{-115}$ & Yes & 51 \\
\hline rs2954029 & TRIBI & 8 & $\mathrm{~A}(0.47)$ & $3 \times 10^{-55}$ & Yes & 51 \\
\hline rs|076|73| & $J M J D I C$ & 10 & $\mathrm{~A}(0.43)$ & $3 \times 10^{-12}$ & No & 51 \\
\hline rs2068888 & CYP26AI & 10 & $\mathrm{G}(0.46)$ & $2 \times 10^{-8}$ & No & 51 \\
\hline rs 174546 & FADSI-2-3 & 11 & $C(0.34)$ & $5 \times 10^{-24}$ & No & 51 \\
\hline rs964I84 & APOAI & II & $C(0.13)$ & $7 \times 10^{-240}$ & Yes & 51 \\
\hline rsII613352 & LRPI & 12 & $C(0.23)$ & $4 \times 10^{-10}$ & No & 51 \\
\hline rs24I2710 & CAPN3 & 15 & $\mathrm{G}(0.02)$ & $2 \times 10^{-8}$ & No & 51 \\
\hline rs2929282 & FRMD5 & 15 & $A(0.05)$ & $2 \times 10^{-11}$ & No & 51 \\
\hline rsII649653 & CTFI & 16 & $C(0.40)$ & $3 \times 10^{-8}$ & No & 51 \\
\hline rs43940I & APOE & 19 & $C(0.36)$ & $1 \times 10^{-30}$ & No & 51 \\
\hline rs575693I & PLA2G6 & 22 & $\mathrm{~T}(0.40)$ & $4 \times 10^{-8}$ & No & 51 \\
\hline \multicolumn{7}{|c|}{ Low-density lipoprotein } \\
\hline rs2479409 & PCSK9 & I & $\mathrm{A}(0.23)$ & $2 \times 10^{-28}$ & No & 51 \\
\hline rs62930I & SORTI & I & $\mathrm{T}(0.22)$ & $1 \times 10^{-170}$ & Yes & 51 \\
\hline rsI367II7 & $A P O B$ & 2 & $G(0.30)$ & $4 \times 10^{-114}$ & Yes & 51 \\
\hline rs499376 & $A B C G 5 / 8$ & 2 & $\mathrm{~T}(0.30)$ & $2 \times 10^{-47}$ & Yes & 51 \\
\hline rs3757354 & MYLIP & 6 & $C(0.22)$ & $1 \times 10^{-11}$ & No & 51 \\
\hline rs 1800562 & HFE & 6 & $\mathrm{G}(0.06)$ & $6 \times 10^{-10}$ & No & 51 \\
\hline rsI564348 & $L P A$ & 6 & $\mathrm{~T}(0.17)$ & $2 \times 10^{-17}$ & Yes & 51 \\
\hline rsIII3634I & PLECI & 8 & $\mathrm{~A}(0.40)$ & $4 \times 10^{-13}$ & No & 51 \\
\hline rs94II489 & $A B O$ & 9 & $C(0.20)$ & $6 \times 10^{-13}$ & Yes & 51 \\
\hline rsII 220462 & ST3GAL4 & II & $\mathrm{G}(0.14)$ & $1 \times 10^{-15}$ & No & 51 \\
\hline rs80I7377 & NYNRIN & 14 & $\mathrm{G}(0.47)$ & $5 \times 10^{-11}$ & No & 51 \\
\hline rs720697I & OSBPL7 & 17 & G (0.49) & $2 \times 10^{-8}$ & No & 51 \\
\hline rs65II720 & $L D L R$ & 19 & $G(0.1 I)$ & $4 \times 10^{-117}$ & Yes & 51 \\
\hline rs4420638 & APOE & 19 & $A(0.17)$ & $9 \times 10^{-147}$ & No & 51 \\
\hline rs6029526 & TOPI & 20 & $\mathrm{~T}(0.47)$ & $4 \times 10^{-19}$ & No & 51 \\
\hline \multicolumn{7}{|c|}{ High-density lipoprotein } \\
\hline rs4660293 & PABPC4 & I & $A(0.23)$ & $4 \times 10^{-10}$ & No & 51 \\
\hline rs 1689800 & ZNF648 & I & $A(0.35)$ & $3 \times 10^{-10}$ & No & 51 \\
\hline rs4846914 & GALNT2 & I & $\mathrm{A}(0.40)$ & $4 \times 10^{-21}$ & No & 51 \\
\hline rs2972I46 & IRSI & 2 & $\mathrm{~T}(0.37)$ & $3 \times 10^{-9}$ & No & 51 \\
\hline rs 12328675 & COBLLI & 2 & $\mathrm{~T}(0.13)$ & $3 \times 10^{-10}$ & No & 51 \\
\hline rs|3|07325 & SLC39A8 & 4 & $C(0.07)$ & $7 \times 10^{-11}$ & No & 51 \\
\hline rs6450I76 & $A R L I 5$ & 5 & $\mathrm{G}(0.26)$ & $5 \times 10^{-8}$ & No & 51 \\
\hline rs28I4944 & C6orflo6 & 6 & $G(0.16)$ & $4 \times 10^{-9}$ & No & 51 \\
\hline rs605066 & CITED2 & 6 & $\mathrm{~T}(0.42)$ & $3 \times 10^{-8}$ & No & 51 \\
\hline rs $108465 \mid$ & $\angle P A$ & 6 & $G(0.16)$ & $3 \times 10^{-8}$ & Yes & 51 \\
\hline rs2293889 & TRPSI & 8 & $\mathrm{G}(0.4 \mathrm{I})$ & $6 \times 10^{-11}$ & No & 51 \\
\hline rs473I702 & $K L F / 4$ & 7 & $C(0.48)$ & $1 \times 10^{-15}$ & No & 51 \\
\hline rs9987289 & PPPIR3B & 8 & G (0.09) & $6 \times 10^{-25}$ & No & 51 \\
\hline
\end{tabular}


Table 4 (Continued)

\begin{tabular}{|c|c|c|c|c|c|c|c|}
\hline rsID & Loci & Chr & $\begin{array}{l}\text { Risk allele (Risk } \\
\text { allele frequency) }\end{array}$ & \multicolumn{2}{|c|}{ Combined $P$-value } & $\begin{array}{l}\text { Known effect } \\
\text { on CAD }\end{array}$ & Reference \\
\hline rs581080 & TTC39B & 9 & $C(0.18)$ & \multicolumn{2}{|l|}{$3 \times 10^{-12}$} & No & 51 \\
\hline rsl883025 & $A B C A I$ & 9 & $C(0.25)$ & \multicolumn{2}{|l|}{$2 \times 10^{-33}$} & No & 51 \\
\hline rs2923084 & AMPD3 & 11 & $A(0.17)$ & \multicolumn{2}{|l|}{$5 \times 10^{-8}$} & No & 51 \\
\hline rs313644I & LRP4 & II & $\mathrm{T}(0.15)$ & \multicolumn{2}{|l|}{$3 \times 10^{-18}$} & No & 51 \\
\hline rs7134594 & PDE3A & 12 & $C(0.42)$ & \multicolumn{2}{|l|}{$4 \times 10^{-8}$} & No & 51 \\
\hline rs7।34594 & $M V K$ & 12 & $\mathrm{~T}(0.47)$ & \multicolumn{2}{|l|}{$7 \times 10^{-15}$} & No & 51 \\
\hline rs4759375 & SBNOI & 12 & $C(0.06)$ & \multicolumn{2}{|l|}{$7 \times 10^{-9}$} & No & 51 \\
\hline rs4765I 27 & ZNF664 & 12 & $\mathrm{G}(0.34)$ & \multicolumn{2}{|l|}{$3 \times 10^{-10}$} & No & 51 \\
\hline rs838880 & SCARBI & 12 & $\mathrm{~T}(0.3 \mathrm{I})$ & \multicolumn{2}{|l|}{$3 \times 10^{-14}$} & No & 51 \\
\hline rsI532085 & LIPC & 15 & G (0.39) & \multicolumn{2}{|l|}{$3 \times 10^{-96}$} & No & 51 \\
\hline rs2652834 & LACTB & 15 & $\mathrm{G}(0.20)$ & \multicolumn{2}{|l|}{$9 \times 10^{-9}$} & No & 51 \\
\hline rs376426I & CETP & 16 & $C(0.32)$ & \multicolumn{2}{|l|}{$7 \times 10^{-380}$} & No & 51 \\
\hline rs 16942887 & LCAT & 16 & $\mathrm{G}(0.12)$ & \multicolumn{2}{|l|}{$8 \times 10^{-33}$} & No & 51 \\
\hline rs2925979 & CMIP & 16 & $C(0.30)$ & \multicolumn{2}{|l|}{$2 \times 10^{-11}$} & No & 51 \\
\hline rsII869286 & STARD3 & 17 & $C(0.34)$ & \multicolumn{2}{|l|}{$1 \times 10^{-13}$} & No & 51 \\
\hline rs4l48008 & $A B C A 8$ & 17 & $C(0.32)$ & \multicolumn{2}{|l|}{$2 \times 10^{-10}$} & No & 51 \\
\hline rs4129767 & PGSI & 17 & $\mathrm{~A}(0.49)$ & \multicolumn{2}{|l|}{$8 \times 10^{-9}$} & No & 51 \\
\hline rs7241918 & LIPG & 18 & $\mathrm{~T}(0.17)$ & \multicolumn{2}{|l|}{$3 \times 10^{-49}$} & No & 51 \\
\hline rs $12967 \mid 35$ & $M C 4 R$ & 18 & $\mathrm{G}(0.23)$ & $7 \times 10^{-9}$ & & No & 51 \\
\hline rs7255436 & ANGPTL4 & 19 & $\mathrm{~A}(0.47)$ & $3 \times 10^{-8}$ & & No & 51 \\
\hline rs737337 & LOC55908 & 19 & $\mathrm{~T}(0.08)$ & $3 \times 10^{-9}$ & & No & 51 \\
\hline rs386000 & LILRA3 & 19 & $\mathrm{G}(0.20)$ & $4 \times 10^{-19}$ & & No & 51 \\
\hline rsl80096I & HNF4A & 20 & $C(0.03)$ & $1 \times 10^{-15}$ & & No & 51 \\
\hline rs6065906 & PLTP & 20 & $\mathrm{~T}(0.18)$ & $2 \times 10^{-22}$ & & No & 51 \\
\hline rs 181362 & UBE2L3 & 22 & $C(0.20)$ & $1 \times 10^{-8}$ & & No & 51 \\
\hline rsID & Loci & Chr & Risk allele (Risk & $P$-value & & Known effect & Reference \\
\hline & & & allele frequency) & SBP & DBP & On CAD & \\
\hline Blood pressure & & & & & & & \\
\hline rs2932538 & MOVIO & I & $\mathrm{G}(0.75)$ & $1.2 \times 10^{-9}$ & $9.9 \times 10^{-10}$ & No & 48 \\
\hline rs 17367504 & MTHFR-NPPB & 1 & $\mathrm{G}(0.15)$ & $8.7 \times 10^{-22}$ & $3.5 \times 10^{-19}$ & No & 48 \\
\hline rs 13002573 & FIGN & 2 & $\mathrm{G}(0.20)$ & $3.25 \times 10^{-7}$ & $4.02 \times 10^{-2}$ & No & 53 \\
\hline rsI446468 & FIGN & 2 & $\mathrm{~T}(0.53)$ & $1.82 \times 10^{-12}$ & $6.88 \times 10^{-9}$ & No & 53 \\
\hline rs319690 & MAP4 & 3 & $\mathrm{~T}(0.5 \mathrm{I})$ & $4.74 \times 10^{-8}$ & $1.84 \times 10^{-8}$ & No & 53 \\
\hline rs |30827|I & SLC4A7 & 3 & $\mathrm{~T}(0.78)$ & $1.5 \times 10^{-6}$ & $3.8 \times 10^{-9}$ & No & 48 \\
\hline rs419076 & MECOM & 3 & $\mathrm{~T}(0.47)$ & $1.80 \times 10^{-13}$ & $2.1 \times 10^{-12}$ & No & 48 \\
\hline rs3774372 & ULK4 & 3 & $\mathrm{~T}(0.83)$ & 0.39 & $9.0 \times 10^{-14}$ & No & 48 \\
\hline rs87/606 & $\mathrm{CHIC2}$ & 4 & $\mathrm{~T}(0.86)$ & $3.04 \times 10^{-4}$ & $8.85 \times 10^{-1}$ & No & 53 \\
\hline rs|3107325 & SLC39A8 & 4 & $\mathrm{~T}(0.05)$ & $3.3 \times 10^{-14}$ & $2.3 \times 10^{-17}$ & No & 48 \\
\hline rs|3|3957| & GUCYIA3-GUCYIB3 & 4 & $C(0.76)$ & $1.2 \times 10^{-6}$ & $2.2 \times 10^{-10}$ & Yes & 48 \\
\hline rs|458038 & FGF5 & 4 & $\mathrm{~T}(0.29)$ & $1.5 \times 10^{-23}$ & $8.5 \times 10^{-25}$ & No & 48 \\
\hline rsII7377I & NPR3-C5orf23 & 5 & $G(0.60)$ & $1.8 \times 10^{-16}$ & $9.1 \times 10^{-12}$ & No & 48 \\
\hline rsII953630 & EBFI & 5 & $\mathrm{~T}(0.37)$ & $3.0 \times 10^{-11}$ & $3.8 \times 10^{-13}$ & No & 48 \\
\hline rsl799945 & HFE & 6 & $G(0.14)$ & $7.7 \times 10^{-12}$ & $1.5 \times 10^{-15}$ & No & 48 \\
\hline rs805303 & BAT2-BAT5 & 6 & $G(0.6 I)$ & $1.5 \times 10^{-11}$ & $4.4 \times 10^{-10}$ & No & 48 \\
\hline rs I7477|77 & PIK3CG & 7 & $\mathrm{~T}(0.72)$ & $5.67 \times 10^{-11}$ & $1.40 \times 10^{-1}$ & No & 53 \\
\hline rs207I5I8 & NOV & 8 & $\mathrm{~T}(0.17)$ & $2.08 \times 10^{-2}$ & $3.89 \times 10^{-3}$ & No & 53 \\
\hline rs 2782980 & $A D R B I$ & 10 & $\mathrm{~T}(0.20)$ & $7.66 \times 10^{-7}$ & $9.60 \times 10^{-8}$ & No & 53 \\
\hline rs 4373814 & CACNB2 & 10 & G (0.55) & $4.8 \times 10^{-11}$ & $4.4 \times 10^{-10}$ & No & 48 \\
\hline rs932764 & PLCEI & 10 & $\mathrm{G}(0.44)$ & $7.1 \times 10^{-16}$ & $8.1 \times 10^{-7}$ & No & 48 \\
\hline rsl813353 & CACNB2 & 10 & $\mathrm{~T}(0.68)$ & $2.6 \times 10^{-12}$ & $2.3 \times 10^{-15}$ & No & 48 \\
\hline rs45908I7 & ClOorflo7 & 10 & $\mathrm{G}(0.84)$ & $4.0 \times 10^{-12}$ & $1.3 \times 10^{-12}$ & No & 48 \\
\hline rsIII91548 & CYPI 7AI-NT5C2 & 10 & $\mathrm{~T}(0.91)$ & $6.9 \times 10^{-26}$ & $9.4 \times 10^{-13}$ & Yes & 48 \\
\hline
\end{tabular}


Table 4 (Continued)

\begin{tabular}{|c|c|c|c|c|c|c|c|}
\hline \multirow[t]{2}{*}{ rsID } & \multirow[t]{2}{*}{ Loci } & \multirow[t]{2}{*}{ Chr } & \multirow{2}{*}{$\begin{array}{l}\text { Risk allele (Risk } \\
\text { allele frequency) }\end{array}$} & \multicolumn{2}{|l|}{$P$-value } & \multirow{2}{*}{$\begin{array}{l}\text { Known effect } \\
\text { On CAD }\end{array}$} & \multirow[t]{2}{*}{ Reference } \\
\hline & & & & SBP & DBP & & \\
\hline rsI I 222084 & ADAMTS8 & II & $\mathrm{T}(0.38)$ & $4.00 \times 10^{-4}$ & $3.44 \times 10^{-2}$ & No & 53 \\
\hline rs7129220 & $A D M$ & II & $\mathrm{G}(0.89)$ & $3.0 \times 10^{-12}$ & $6.4 \times 10^{-8}$ & No & 48 \\
\hline rs633185 & FLJ328/0-TMEM I 33 & II & $\mathrm{G}(0.28)$ & $1.2 \times 10^{-17}$ & $2.0 \times 10^{-15}$ & No & 48 \\
\hline rs381815 & PLEKHA7 & II & $\mathrm{T}(0.26)$ & $5.3 \times 10^{-11}$ & $5.3 \times 10^{-10}$ & No & 48 \\
\hline rsI7249754 & ATP2BI & 12 & $\mathrm{G}(0.84)$ & $1.8 \times 10^{-18}$ & $1.2 \times 10^{-14}$ & No & 48 \\
\hline rs3184504 & $\mathrm{SH} 2 \mathrm{~B} 3$ & 12 & $\mathrm{~T}(0.47)$ & $3.8 \times 10^{-18}$ & $3.6 \times 10^{-25}$ & Yes & 48 \\
\hline rsI08504 II & $T B \times 5-T B X 3$ & 12 & $\mathrm{~T}(0.70)$ & $5.4 \times 10^{-8}$ & $5.4 \times 10^{-10}$ & No & 48 \\
\hline rs252I50I & FURIN-FES & 15 & $\mathrm{~T}(0.3 \mathrm{I})$ & $5.2 \times 10^{-19}$ & $1.9 \times 10^{-15}$ & Yes & 48 \\
\hline rsl 378942 & CYPIAI-ULK3 & 15 & $C(0.35)$ & $5.7 \times 10^{-23}$ & $2.7 \times 10^{-26}$ & No & 48 \\
\hline rsI 7608766 & GOSR2 & 17 & $\mathrm{~T}(0.86)$ & $1.1 \times 10^{-10}$ & 0.017 & No & 48 \\
\hline rs I 2940887 & ZNF652 & 17 & $\mathrm{~T}(0.38)$ & $1.8 \times 10^{-10}$ & $2.3 \times 10^{-14}$ & No & 48 \\
\hline rs|327235 & JAGI & 20 & $\mathrm{G}(0.46)$ & $1.9 \times 10^{-8}$ & $1.4 \times 10^{-15}$ & No & 48 \\
\hline rs60I5450 & GNAS-EDN3 & 20 & $\mathrm{G}(0.12)$ & $3.9 \times 10^{-23}$ & $5.6 \times 10^{-23}$ & No & 48 \\
\hline
\end{tabular}

Abbreviations: Chr, chromosome; CAD, coronary artery disease; SBP, systolic blood pressure; DBP, diastolic blood pressure.

is possible to determine a person's genetic risk profile before the development of an adverse cardiovascular risk factor profile, which would allow primary prevention measures to be undertaken earlier in life. ${ }^{2,63}$ Another advantage is the lower cost and higher replicability of genotyping compared with other cardiovascular risk factors. Among the limitations, the small effect sizes of known variants are most notable, despite the highly statistically significant associations between these variants and CAD risk.

Several studies have evaluated the effects on the predictive capacity of classical risk functions when genetic factors are taken into account. While most of the studies have found that these genetic variants (usually expressed as a single variable corresponding to the number of risk alleles carried, known as a genetic risk score) are associated with risk of future CAD events, they have not been found to improve the ability to discriminate between those individuals at particular risk who will develop the disease, although they do improve the reclassification of individuals into more appropriate risk categories, especially those at intermediate risk (Table 5).

\section{Pharmacogenomics}

Pharmacogenomics is the study of the relationship between genetic variability and a patient's response to drug treatment, ie, the efficacy of the drug and/or its adverse effects. ${ }^{64-68}$ Candidate gene and GWAS approaches have been used to identify genetic variants associated with variability in drug response, including several examples in the cardiovascular field, ${ }^{69,70}$ the majority of which have focused on statins, antiplatelet drugs, oral anticoagulants, or beta-blockers. The case of statins and the antiplatelet agent clopidogrel provide two interesting examples in this area.
Statins are widely prescribed to reduce plasma cholesterol levels and cardiovascular risk, and although the majority of patients show a $30 \%-50 \%$ reduction in LDL cholesterol, high interindividual variability is observed. ${ }^{71}$ Several genetic variants in the $H M G C R, A P O E, C E T P$, and $C L M N$ genes have been reported to be associated with this interindividual variability, but the results have been discordant. ${ }^{69,70}$ Similarly, a variant in the KIF6 gene has been reported to modulate the effect of statins on clinical outcome,,$^{72,73}$ but recent studies have not corroborated this finding. ${ }^{74,75}$ Finally, more than one variant in the $S L C O 1 B 1$ gene is consistently associated with the risk of simvastatin-induced myopathy, with an odds ratio $>4.69,76$

Our second example concerns the prodrug clopidogrel, which is converted into an active metabolite that selectively and irreversibly binds to the P2Y12 receptor on the platelet membrane. Conversion is achieved by the hepatic cytochrome P450 system in a two-step oxidative process, and cytochrome P450 2C19 is involved in both of these steps. The response to treatment with clopidogrel varies markedly between individuals, and the causes of a poor response are not clearly understood, but have been suggested to be related to clinical, cellular, or genetic factors. ${ }^{66,77,78}$ In March 2010, the US Food and Drug Administration added a "boxed warning" to the labeling of clopidogrel, including a reference to patients who do not effectively metabolize the drug and therefore may not receive the full benefits on the basis of their genetic characteristics. ${ }^{79}$ Recently, the American College of Cardiology Foundation and the American Heart Association have published a consensus document addressing this US Food and Drug Administration warning, ${ }^{80}$ stating that the role of genetic tests and the clinical implications and consequences 


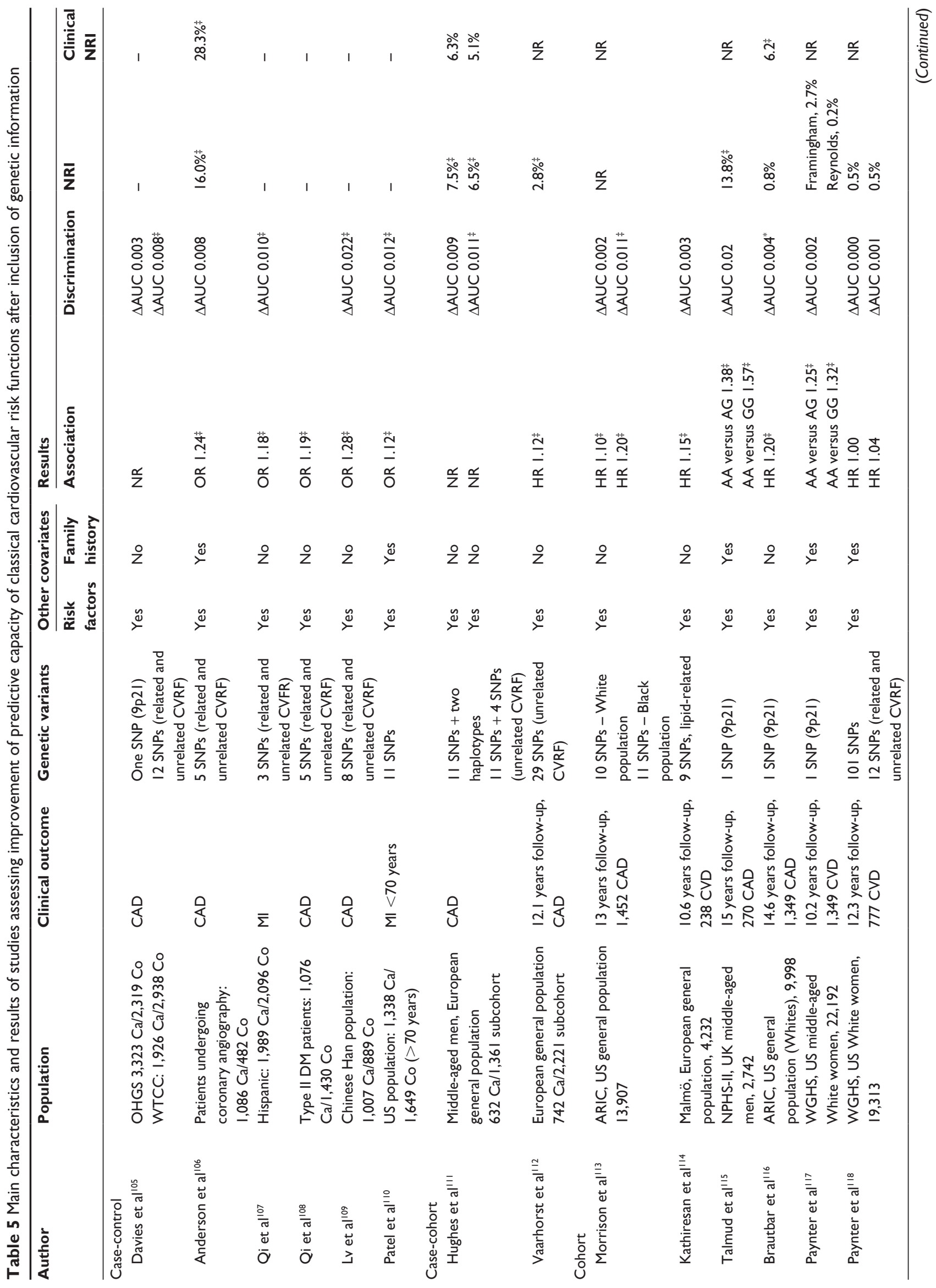




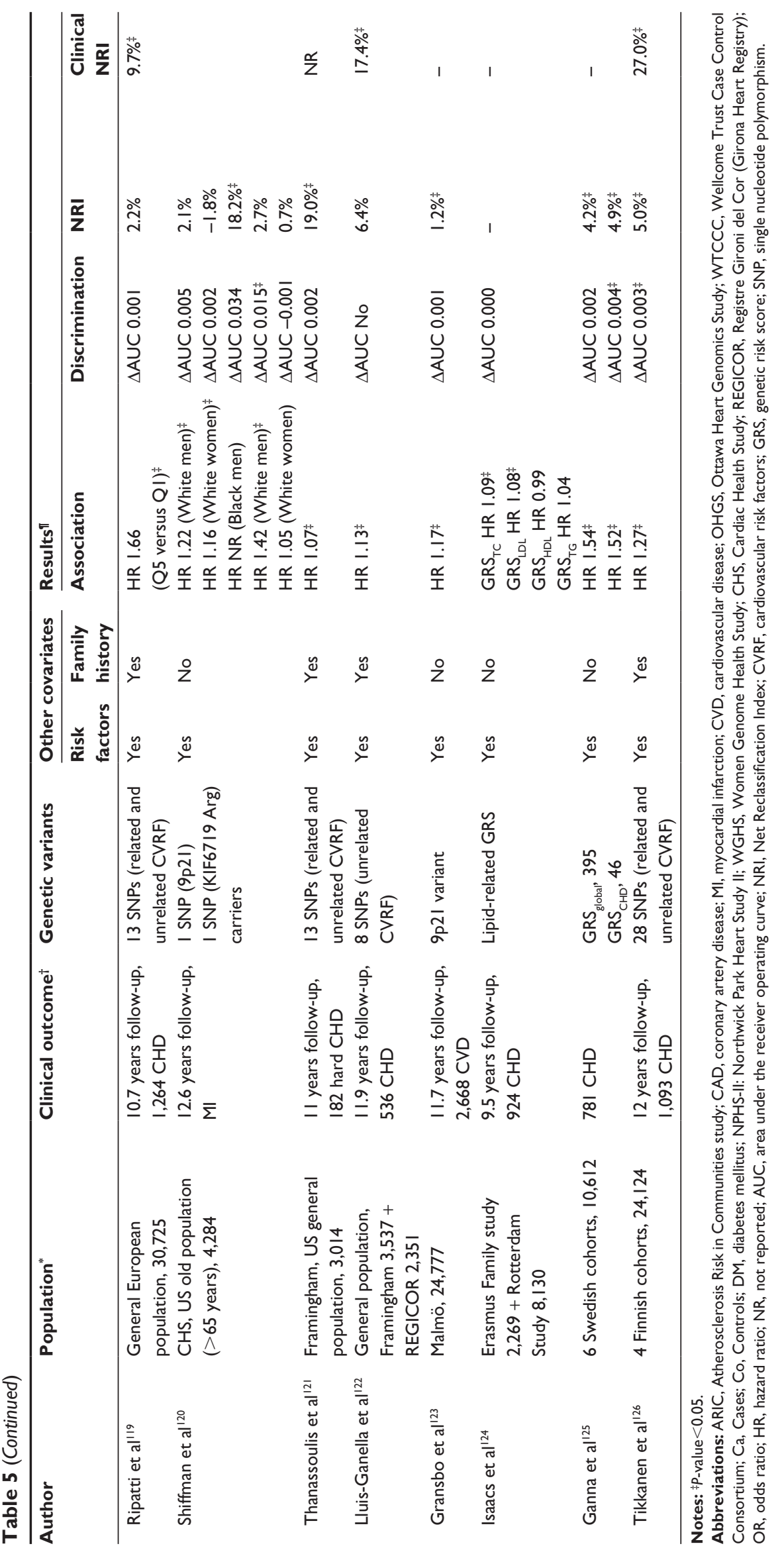


of this testing remain to be determined. Moreover, three recent meta-analyses question the validity of this warning based on the fact that the reported associations are mainly driven by studies with small sample sizes, ${ }^{78,81,82}$ thus, they concluded that current evidence does not support the use of individualized clopidogrel regimens guided by the CYP2C19 genotype.

\section{Conclusion}

In the past 7 years, GWAS have contributed substantially to our understanding of the genetic architecture of complex diseases, including CAD. To date, approximately 40 unique loci have been found to be robustly associated with disease risk in large samples from several populations, a much higher number than those identified by linkage and candidate gene association studies. However, these variants explain only a small proportion of the heritability of CAD. ${ }^{40}$ Additional efforts to improve the analysis strategies, including new imputation and meta-analytic methods, analysis of gene-gene and gene-environment interactions, the integration of different omics, and use of sequencing technologies, are being performed. ${ }^{83-85}$

Although it is not yet clear if or how all of this information on the genetic architecture of CAD can be translated into clinical practice, ${ }^{86}$ we already have some exciting examples of its potential utility. To identify new therapeutic targets, we must first make the difficult transition from the statistical associations reported in GWAS to the functional mechanisms behind these associations. Research on the use of genetic information to improve cardiovascular risk estimation in individuals at intermediate risk can be carried out as a second step or in parallel, and further studies to develop new ways to include this information in risk functions, to evaluate its cost-effectiveness, and to explore the ethical issues are also warranted. ${ }^{87-89}$ Finally, although medicine is always a "personalized science and art", use of genetic information to identify the most effective and least harmful drug for each patient is also a goal of so-called genetic personalized medicine.

\section{Acknowledgments}

This work was supported by the Spanish Ministry of Science and Innovation through the Carlos III Health Institute (Red de Investigación Cardiovascular; Programa HERACLES RD12/0042), Health Research Fund (FIS-PI09/90506), and the Government of Catalunya through the Agency for Management of University and Research Grants (2009 SGR 1195). We acknowledge the contribution of ThePaperMill (http://www.thepapermill.cat) to the critical reading and English language review of this paper.

\section{Disclosure}

The authors report no other conflicts of interest in this work.

\section{References}

1. Murray CJ, Vos T, Lozano R, et al. Disability-adjusted life years (DALYs) for 291 diseases and injuries in 21 regions, 1990-2010: a systematic analysis for the Global Burden of Disease Study 2010. Lancet. 2012;380:2197-2223.

2. Lluis-Ganella C. Genetic Factors Associated with Coronary Heart Disease and Analysis of their Predictive Capacity. Barcelona, Spain: Universitat Pompeu Fabra; 2012.

3. Mecchia D, Lavezzi AM, Mauri M, Matturri L. Feto-placental atherosclerotic lesions in intrauterine fetal demise: role of parental cigarette smoking. Open Cardiovasc Med J. 2009;3:51-56.

4. Lusis AJ, Mar R, Pajukanta P. Genetics of atherosclerosis. Annu Rev Genomics Hum Genet. 2004;5:189-218.

5. Glass CK, Witztum JL. Atherosclerosis. the road ahead. Cell. 2001;104:503-516.

6. Sanz J, Moreno P, Fuster V. The year in atherothrombosis. J Am Coll Cardiol. 2013;24;62:1131-1143.

7. Tabas I, Glass CK. Anti-inflammatory therapy in chronic disease: challenges and opportunities. Science. 2013;339:166-172.

8. Sakakura K, Nakano M, Otsuka F, Ladich E, Kolodgie FD, Virmani R. Pathophysiology of atherosclerosis plaque progression. Heart Lung Circ. 2013;22:399-411.

9. Ghattas A, Griffiths HR, Devitt A, Lip GY, Shantsila E. Monocytes in coronary artery disease and atherosclerosis: where are we now? $\mathrm{J} \mathrm{Am}$ Coll Cardiol. 2013;62:1541-1551.

10. Libby P, Ridker PM, Hansson GK. Progress and challenges in translating the biology of atherosclerosis. Nature. 2011;473:317-325.

11. Witztum JL, Lichtman AH. The influence of innate and adaptive immune responses on atherosclerosis. Annu Rev Pathol. August 7, 2013. [Epub ahead of print.]

12. Libby P. Inflammation in atherosclerosis. Arterioscler Thromb Vasc Biol. 2012;32:2045-2051.

13. Tabas I. Macrophage death and defective inflammation resolution in atherosclerosis. Nat Rev Immunol. 2010;10:36-46.

14. Finn AV, Nakano M, Narula J, Kolodgie FD, Virmani R. Concept of vulnerable/unstable plaque. Arterioscler Thromb Vasc Biol. 2010;30: $1282-1292$.

15. Doyle B, Caplice N. Plaque neovascularization and antiangiogenic therapy for atherosclerosis. J Am Coll Cardiol. 2007;49: 2073-2080.

16. Visscher PM, Hill WG, Wray NR. Heritability in the genomics era - concepts and misconceptions. Nat Rev Genet. 2008;9: 255-266.

17. Elosua R, Lluis-Ganella C, Lucas G. Research into the genetic component of heart disease: from linkage studies to genome-wide genotyping. Rev Esp Cardiol Suppl. 2009;9 Suppl:24B-38B.

18. Peden JF, Farrall M. Thirty-five common variants for coronary artery disease: the fruits of much collaborative labour. Hum Mol Genet 2011;20:R198-R205.

19. Chial H. Mendelian genetics: patterns of inheritance and single-gene disorders. Nature Education. 2008;1(1). Available from: http:// www.nature.com/scitable/topicpage/mendelian-genetics-patterns-ofinheritance-and-single-966. Accessed November 4, 2013.

20. Lobo I. Multifactorial inheritance and genetic disease. Nature Education 2008;1(1). Available from: http://www.nature.com/scitable/topicpage/ multifactorial-inheritance-and-genetic-disease-919. Accessed November 4, 2013.

21. Deloukas P, Kanoni S, Willenborg C, et al. Large-scale association analysis identifies new risk loci for coronary artery disease. Nat Genet. 2013;45:25-33. 
22. Dawn TM, Barrett JH. Genetic linkage studies. Lancet. 2005;366: 1036-1044.

23. Kathiresan S, Srivastava D. Genetics of human cardiovascular disease. Cell. 2012;148:1242-1257.

24. Wang JG, Staessen JA, Franklin SS, Fagard R, Gueyffier F. Systolic and diastolic blood pressure lowering as determinants of cardiovascular outcome. Hypertension. 2005;45:907-913.

25. Helgadottir A, Manolescu A, Thorleifsson G, et al. The gene encoding 5-lipoxygenase activating protein confers risk of myocardial infarction and stroke. Nat Genet. 2004;36:233-239.

26. Wang L, Fan C, Topol SE, Topol EJ, Wang Q. Mutation of MEF2A in an inherited disorder with features of coronary artery disease. Science. 2003;302:1578-1581.

27. Varret M, Rabes JP, Saint-Jore B, et al. A third major locus for autosomal dominant hypercholesterolemia maps to 1p34.1-p32. Am J Hum Genet. 1999;64:1378-1387.

28. Risch N, Merikangas K. The future of genetic studies of complex human diseases. Science. 1996;273:1516-1517.

29. Zhu M, Zhao S. Candidate gene identification approach: progress and challenges. Int J Biol Sci. 2007;3:420-427.

30. Tabor HK, Risch NJ, Myers RM. Candidate-gene approaches for studying complex genetic traits: practical considerations. Nat Rev Genet. 2002;3:391-397.

31. Attia J, Ioannidis JP, Thakkinstian A, et al. How to use an article about genetic association. $\mathrm{C}$ : What are the results and will they help me in caring for my patients? JAMA. 2009;301:304-308.

32. Lohmueller KE, Pearce CL, Pike M, Lander ES, Hirschhorn JN. Meta-analysis of genetic association studies supports a contribution of common variants to susceptibility to common disease. Nat Genet. 2003;33:177-182.

33. Visscher PM, Brown MA, McCarthy MI, Yang J. Five years of GWAS discovery. Am J Hum Genet. 2012;90:7-24.

34. The International HapMap Consortium. A haplotype map of the human genome. Nature. 2005;437:1299-1320.

35. US Department of Energy Human Genome Project. Available from: http://www.ornl.gov/hgmis. Accessed November 4, 2013.

36. Arking DE, Chakravarti A. Understanding cardiovascular disease through the lens of genome-wide association studies. Trends Genet. 2009;25:387-394.

37. Purcell S, Neale B, Todd-Brown K, et al. PLINK: a tool set for wholegenome association and population-based linkage analyses. Am J Hum Genet. 2007;81:559-575.

38. Aulchenko YS, Ripke S, Isaacs A, van Duijn CM. GenABEL: an Rlibrary for genome-wide association analysis. Bioinformatics. 2007;23:1294-1296.

39. Roberts R, Stewart AF. Genes and coronary artery disease: where are we? J Am Coll Cardiol. 2012;60:1715-1721.

40. Manolio TA, Collins FS, Cox NJ, et al. Finding the missing heritability of complex diseases. Nature. 2009;461:747-753.

41. Hindorff LA, Junkins HA, Mehta JP, Manolio TA. A catalog of published genome-wide association studies. Available from: http:// www.genome.gov/gwastudies. Accessed November 4, 2013.

42. Marian AJ. Challenges in medical applications of whole exome/genome sequencing discoveries. Trends Cardiovasc Med. 2012;22:219-223.

43. Almasy L. The role of phenotype in gene discovery in the whole genome sequencing era. Hum Genet. 2012;131:1533-1540.

44. Helgadottir A, Thorleifsson G, Manolescu A, et al. A common variant on chromosome 9p21 affects the risk of myocardial infarction. Science. 2007;316:1491-1493.

45. McPherson R, Pertsemlidis A, Kavaslar N, et al. A common allele on chromosome 9 associated with coronary heart disease. Science. 2007;316:1488-1491.

46. Wellcome Trust Case Control Consortium. Genome-wide association study of 14,000 cases of seven common diseases and 3,000 shared controls. Nature. 2007;447:661-678.
47. Dupuis J, Langenberg C, Prokopenko I, et al. New genetic loci implicated in fasting glucose homeostasis and their impact on type 2 diabetes risk. Nat Genet. 2010;42:105-116.

48. Ehret GB, Munroe PB, Rice KM, et al. Genetic variants in novel pathways influence blood pressure and cardiovascular disease risk. Nature. 2011;478:103-109.

49. Heid IM, Jackson AU, Randall JC, et al. Meta-analysis identifies 13 new loci associated with waist-hip ratio and reveals sexual dimorphism in the genetic basis of fat distribution. Nat Genet. 2010;42:949-960.

50. Speliotes EK, Willer CJ, Berndt SI, et al. Association analyses of 249,796 individuals reveal 18 new loci associated with body mass index. Nat Genet. 2010;42:937-948.

51. Teslovich TM, Musunuru K, Smith AV, et al. Biological, clinical and population relevance of 95 loci for blood lipids. Nature. 2010;466: 707-713.

52. Voight BF, Scott LJ, Steinthorsdottir V, et al. Twelve type 2 diabetes susceptibility loci identified through large-scale association analysis. Nat Genet. 2010;42:579-589.

53. Wain LV, Verwoert GC, O'Reilly PF, et al. Genome-wide association study identifies six new loci influencing pulse pressure and mean arterial pressure. Nat Genet. 2011;43:1005-1011.

54. Abifadel M, Varret M, Rabes JP, et al. Mutations in PCSK9 cause autosomal dominant hypercholesterolemia. Nat Genet. 2003;34:154-156.

55. Lambert G, Sjouke B, Choque B, Kastelein JJ, Hovingh GK. The PCSK9 decade. J Lipid Res. 2012;53:2515-2524.

56. Roth EM, McKenney JM, Hanotin C, Asset G, Stein EA. Atorvastatin with or without an antibody to PCSK9 in primary hypercholesterolemia. N Engl J Med. 2012;367:1891-1900.

57. McPherson R. Chromosome 9p21.3 locus for coronary artery disease: how little we know. J Am Coll Cardiol. 2013;62(15):1382-1383.

58. Aguero F, Degano IR, Subirana I, et al. Impact of a partial smoke-free legislation on myocardial infarction incidence, mortality and casefatality in a population-based registry: the REGICOR study. PLoS One. 2013;8:e53722.

59. Perk J, De Backer G, Gohlke H, et al. European guidelines on cardiovascular disease prevention in clinical practice (version 2012). Rev Esp Cardiol. 2012;65:66-241.

60. Expert Panel on Detection, Evaluation, and Treatment of High Blood Cholesterol in Adults. Executive Summary of The Third Report of The National Cholesterol Education Program (NCEP) Expert Panel on Detection, Evaluation, And Treatment of High Blood Cholesterol In Adults (Adult Treatment Panel III). JAMA. 2001;285:2486-2497.

61. Wilson PW, D’Agostino RB, Levy D, Belanger AM, Silbershatz H, Kannel WB. Prediction of coronary heart disease using risk factor categories. Circulation. 1998;97:1837-1847.

62. Marrugat J, Vila J, Baena-Diez JM, et al. Relative validity of the 10-year cardiovascular risk estimate in a population cohort of the REGICOR study. Rev Esp Cardiol. 2011;64:385-394.

63. Wang TJ. Assessing the role of circulating, genetic, and imaging biomarkers in cardiovascular risk prediction. Circulation. 2011;123: 551-565.

64. Candore G, Balistreri CR, Caruso M, et al. Pharmacogenomics: a tool to prevent and cure coronary heart disease. Curr Pharm Des. 2007; 13:3726-3734.

65. Evans WE, McLeod HL. Pharmacogenomics - drug disposition, drug targets, and side effects. N Engl J Med. 2003;348:538-549.

66. Marin F, Gonzalez-Conejero R, Capranzano P, Bass TA, Roldan V, Angiolillo DJ. Pharmacogenetics in cardiovascular antithrombotic therapy. J Am Coll Cardiol. 2009;54:1041-1057.

67. Motulsky AG. Drug reactions enzymes, and biochemical genetics. J Am Med Assoc. 1957;165:835-837.

68. Roses AD. Pharmacogenetics and future drug development and delivery. Lancet. 2000;355:1358-1361.

69. Voora D, Ginsburg GS. Clinical application of cardiovascular pharmacogenetics. J Am Coll Cardiol. 2012;60:9-20. 
70. Verschuren JJ, Trompet S, Wessels JA, et al. A systematic review on pharmacogenetics in cardiovascular disease: is it ready for clinical application? Eur Heart J. 2012;33:165-175.

71. Simon JA, Lin F, Hulley SB, et al. Phenotypic predictors of response to simvastatin therapy among African-Americans and Caucasians: the Cholesterol and Pharmacogenetics (CAP) Study. Am J Cardiol. 2006;97:843-850.

72. Iakoubova OA, Sabatine MS, Rowland CM, et al. Polymorphism in KIF6 gene and benefit from statins after acute coronary syndromes: results from the PROVE IT-TIMI 22 study. J Am Coll Cardiol. 2008;51: 449-455.

73. Iakoubova OA, Tong $\mathrm{CH}$, Rowland CM, et al. Association of the Trp719Arg polymorphism in kinesin-like protein 6 with myocardial infarction and coronary heart disease in 2 prospective trials: the CARE and WOSCOPS trials. J Am Coll Cardiol. 2008;51:435-443.

74. Hopewell JC, Parish S, Clarke R, et al. No impact of KIF6 genotype on vascular risk and statin response among 18,348 randomized patients in the heart protection study. J Am Coll Cardiol. 2011;57:2000-2007.

75. Ridker PM, MacFadyen JG, Glynn RJ, Chasman DI. Kinesin-like protein 6 (KIF6) polymorphism and the efficacy of rosuvastatin in primary prevention. Circ Cardiovasc Genet. 2011;4:312-317.

76. Link E, Parish S, Armitage J, et al. SLCO1B1 variants and statininduced myopathy - a genomewide study. N Engl J Med. 2008;359: 789-799

77. Johnson JA, Cavallari LH, Beitelshees AL, Lewis JP, Shuldiner AR, Roden DM. Pharmacogenomics: application to the management of cardiovascular disease. Clin Pharmacol Ther. 2011;90:519-531.

78. Zabalza M, Subirana I, Sala J, et al. Meta-analyses of the association between cytochrome CYP2C19 loss- and gain-of-function polymorphisms and cardiovascular outcomes in patients with coronary artery disease treated with clopidogrel. Heart. 2012;98:100-108.

79. US Department of Health and Human Services. Information on clopidogrel bisulfate. 27-10-2007. Available from: http://www.fda.gov/ Drugs/DrugSafety/PostmarketDrugSafetyInformationforPatientsand Providers/ucm190836.htm. Accessed November 4, 2013.

80. Holmes DR Jr, Dehmer GJ, Kaul S, Leifer D, O'Gara PT, Stein CM. ACCF/AHA Clopidogrel clinical alert: approaches to the FDA "boxed warning": a report of the American College of Cardiology Foundation Task Force on Clinical Expert Consensus Documents and the American Heart Association. Circulation. 2010;122:537-557.

81. Bauer T, Bouman HJ, van Werkum JW, Ford NF, ten Berg JM, Taubert D. Impact of CYP2C19 variant genotypes on clinical efficacy of antiplatelet treatment with clopidogrel: systematic review and meta-analysis. BMJ. 2011;343:d4588.

82. Holmes MV, Perel P, Shah T, Hingorani AD, Casas JP. CYP2C19 genotype, clopidogrel metabolism, platelet function, and cardiovascular events: a systematic review and meta-analysis. JAMA. 2011;306:2704-2714.

83. Maouche S, Schunkert H. Strategies beyond genome-wide association studies for atherosclerosis. Arterioscler Thromb Vasc Biol. 2012;32:170-181.

84. Evangelou E, Ioannidis JP. Meta-analysis methods for genome-wide association studies and beyond. Nat Rev Genet. 2013;14:379-389.

85. Prins BP, Lagou V, Asselbergs FW, Snieder H, Fu J. Genetics of coronary artery disease: genome-wide association studies and beyond. Atherosclerosis. 2012;225:1-10.

86. Manolio TA. Bringing genome-wide association findings into clinical use. Nat Rev Genet. 2013;14:549-558

87. Chatterjee N, Wheeler B, Sampson J, Hartge P, Chanock SJ, Park JH. Projecting the performance of risk prediction based on polygenic analyses of genome-wide association studies. Nat Genet. 2013;45: 400-403.

88. Hudson KL, Holohan MK, Collins FS. Keeping pace with the times - the Genetic Information Nondiscrimination Act of 2008. N Engl J Med. 2008;358:2661-2663.
89. Nora JJ, Lortscher RH, Spangler RD, Nora AH, Kimberling WJ. Genetic-epidemiologic study of early-onset ischemic heart disease. Circulation. 1980;61:503-508.

90. Zdravkovic S, Wienke A, Pedersen NL, Marenberg ME, Yashin AI, de Faire U. Heritability of death from coronary heart disease: a 36-year follow-up of 20966 Swedish twins. J Intern Med. 2002;252: 247-254.

91. Wienke A, Holm NV, Skytthe A, Yashin AI. The heritability of mortality due to heart diseases: a correlated frailty model applied to Danish twins. Twin Res. 2001;4:266-274.

92. Peyser PA, Bielak LF, Chu JS, et al. Heritability of coronary artery calcium quantity measured by electron beam computed tomography in asymptomatic adults. Circulation. 2002;106:304-308.

93. Xiang AH, Azen SP, Buchanan TA, et al. Heritability of subclinical atherosclerosis in Latino families ascertained through a hypertensive parent. Arterioscler Thromb Vasc Biol. 2002;22:843-848.

94. Fox CS, Polak JF, Chazaro I, et al. Genetic and environmental contributions to atherosclerosis phenotypes in men and women: heritability of carotid intima-media thickness in the Framingham Heart Study. Stroke. 2003;34:397-401.

95. Swan L, Birnie DH, Inglis G, Connell JM, Hillis WS. The determination of carotid intima medial thickness in adults - a population-based twin study. Atherosclerosis. 2003;166:137-141.

96. North KE, MacCluer JW, Devereux RB, et al. Heritability of carotid artery structure and function: the Strong Heart Family Study. Arterioscler Thromb Vasc Biol. 2002;22:1698-1703.

97. Hunt KJ, Duggirala R, Goring HH, et al. Genetic basis of variation in carotid artery plaque in the San Antonio Family Heart Study. Stroke. 2002;33:2775-2780.

98. Hennekens CH, Buring JE. Epidemiology in Medicine. Boston, MA: Little, Brown, and Company; 1987.

99. Hirschhorn JN, Lohmueller K, Byrne E, Hirschhorn K. A comprehensive review of genetic association studies. Genet Med. 2002;4:45-61.

100. Hulley S, Cummings SR, Browner WS. Designing Clinical Research: An Epidemiologic Approach. Baltimore, MD: Lippincott, Williams and Wilkins; 2001.

101. Khoury MJ, Beaty T, Cohen B. Fundamentals of Genetic Epidemiology. New York, NY: Oxford University Press; 1993.

102. Ioannidis JP. Why most discovered true associations are inflated. Epidemiology. 2008;19:640-648.

103. Ioannidis JP. Why most published research findings are false. PLoS Med. 2005;2:e124.

104. Pearson TA, Manolio TA. How to interpret a genome-wide association study. JAMA. 2008;299:1335-1344.

105. Davies RW, Dandona S, Stewart AF, et al. Improved prediction of cardiovascular disease based on a panel of single nucleotide polymorphisms identified through genome-wide association studies. Circ Cardiovasc Genet. 2010;3:468-474.

106. Anderson JL, Horne BD, Camp NJ, et al. Joint effects of common genetic variants from multiple genes and pathways on the risk of premature coronary artery disease. Am Heart J. 2010;160: 250-256.

107. Qi L, Ma J, Qi Q, Hartiala J, Allayee H, Campos H. Genetic risk score and risk of myocardial infarction in Hispanics. Circulation. 2011;123: 374-380.

108. Qi L, Parast L, Cai T, et al. Genetic susceptibility to coronary heart disease in type 2 diabetes: 3 independent studies. J Am Coll Cardiol. 2011;58:2675-2682.

109. Lv X, Zhang Y, Rao S, et al. Joint effects of genetic variants in multiple loci on the risk of coronary artery disease in Chinese Han subjects. Circ J. 2012;76:1987-1992.

110. Patel RS, Sun YV, Hartiala J, et al. Association of a genetic risk score with prevalent and incident myocardial infarction in subjects undergoing coronary angiography. Circ Cardiovasc Genet. 2012;5: 441-449. 
111. Hughes MF, Saarela O, Stritzke J, et al. Genetic markers enhance coronary risk prediction in men: the MORGAM prospective cohorts. PLoS One. 2012;7:e40922.

112. Vaarhorst AA, Lu Y, Heijmans BT, et al. Literature-based genetic risk scores for coronary heart disease: the Cardiovascular Registry Maastricht (CAREMA) prospective cohort study. Circ Cardiovasc Genet. 2012;5:202-209.

113. Morrison AC, Bare LA, Chambless LE, et al. Prediction of coronary heart disease risk using a genetic risk score: the Atherosclerosis Risk in Communities study. Am J Epidemiol. 2007;166:28-35.

114. Kathiresan S, Melander O, Anevski D, et al. Polymorphisms associated with cholesterol and risk of cardiovascular events. $N$ Engl J Med. 2008;358:1240-1249.

115. Talmud PJ, Cooper JA, Palmen J, et al. Chromosome 9p21.3 coronary heart disease locus genotype and prospective risk of CHD in healthy middle-aged men. Clin Chem. 2008;54:467-474.

116. Brautbar A, Ballantyne CM, Lawson K, et al. Impact of adding a single allele in the 9p21 locus to traditional risk factors on reclassification of coronary heart disease risk and implications for lipid-modifying therapy in the Atherosclerosis Risk in Communities study. Circ Cardiovasc Genet. 2009;2:279-285.

117. Paynter NP, Chasman DI, Buring JE, Shiffman D, Cook NR, Ridker PM. Cardiovascular disease risk prediction with and without knowledge of genetic variation at chromosome 9p21.3. Ann Intern Med. 2009;150:65-72.

118. Paynter NP, Chasman DI, Pare G, et al. Association between a literature-based genetic risk score and cardiovascular events in women. JAMA. 2010;303:631-637.
119. Ripatti S, Tikkanen E, Orho-Melander M, et al. A multilocus genetic risk score for coronary heart disease: case-control and prospective cohort analyses. Lancet. 2010;376:1393-1400.

120. Shiffman D, O'Meara ES, Rowland CM, et al. The contribution of a 9p21.3 variant, a KIF6 variant, and C-reactive protein to predicting risk of myocardial infarction in a prospective study. BMC Cardiovasc Disord. 2011;11:10.

121. Thanassoulis G, Peloso GM, Pencina MJ, et al. A genetic risk score is associated with incident cardiovascular disease and coronary artery calcium - the Framingham Heart Study. Circ Cardiovasc Genet. 2012;5:113-121.

122. Lluis-Ganella C, Subirana I, Lucas G, et al. Assessment of the value of a genetic risk score in improving the estimation of coronary risk. Atherosclerosis. 2012;222:456-463.

123. Gransbo K, Almgren P, Sjogren M, et al. Chromosome 9p21 genetic variation explains $13 \%$ of cardiovascular disease incidence but does not improve risk prediction. J Intern Med. 2013;274:233-240.

124. Isaacs A, Willems SM, Bos D, et al. Risk scores of common genetic variants for lipid levels influence atherosclerosis and incident coronary heart disease. Arterioscler Thromb Vasc Biol. 2013;33:2233-2239.

125. Ganna A, Magnusson PK, Pedersen NL, et al. Multilocus genetic risk scores for coronary heart disease prediction. Arterioscler Thromb Vasc Biol. 2013;33:2267-2272.

126. Tikkanen E, Havulinna AS, Palotie A, Salomaa V, Ripatti S. Genetic risk prediction and a 2-stage risk screening strategy for coronary heart disease. Arterioscler Thromb Vasc Biol. 2013;33:2261-2266.
The Application of Clinical Genetics

\section{Publish your work in this journal}

The Application of Clinical Genetics is an international, peer-reviewed open access journal that welcomes laboratory and clinical findings in the field of human genetics. Specific topics include: Population genetics; Functional genetics; Natural history of genetic disease; Management of genetic disease; Mechanisms of genetic disease; Counseling and ethical

\section{Dovepress}

issues; Animal models; Pharmacogenetics; Prenatal diagnosis; Dysmorphology. The manuscript management system is completely online and includes a very quick and fair peer-review system, which is all easy to use. Visit http://www.dovepress.com/testimonials.php to read real quotes from published authors. 